\title{
NUMERICAL ANALYSIS OF SOME OPTIMAL CONTROL PROBLEMS GOVERNED BY A CLASS OF QUASILINEAR ELLIPTIC EQUATIONS*
}

\author{
EduARdo CASAS $^{1}$ AND Fredi TrÖLtzsCH ${ }^{2}$
}

\begin{abstract}
In this paper, we carry out the numerical analysis of a distributed optimal control problem governed by a quasilinear elliptic equation of non-monotone type. The goal is to prove the strong convergence of the discretization of the problem by finite elements. The main issue is to get error estimates for the discretization of the state equation. One of the difficulties in this analysis is that, in spite of the partial differential equation has a unique solution for any given control, the uniqueness of a solution for the discrete equation is an open problem.
\end{abstract}

Mathematics Subject Classification. 49M25, 35J60, 35B37, 65N30.

Received December 1st, 2008. Revised December 20, 2009.

Published online August 6, 2010.

\section{INTRODUCTION}

In this paper we will study some aspects of numerical analysis for the optimal control problem

(P) $\left\{\begin{array}{l}\min J(u):=\int_{\Omega} L\left(x, y_{u}(x), u(x)\right) \mathrm{d} x, \\ \alpha \leq u(x) \leq \beta \text { for a.e. } x \in \Omega,\end{array}\right.$

where $y_{u}$ is the solution of the state equation

$$
\left\{\begin{aligned}
-\operatorname{div}[a(x, y(x)) \nabla y(x)]+f(x, y(x)) & =u(x) & & \text { in } \Omega \\
y(x) & =0 & & \text { on } \Gamma .
\end{aligned}\right.
$$

Our main goal is to show the strong convergence of the numerical discretization of this problem by finite elements for the state and different kinds of discretizations for the control. For this purpose, we have to derive

\footnotetext{
Keywords and phrases. Quasilinear elliptic equations, optimal control problems, finite element approximations, convergence of discretized controls.

* The first author was supported by Spanish Ministry of Education and Science under projects MTM2008-04206 and "Ingenio Mathematica (i-MATH)" No. CSD2006-00032 (Consolider Ingenio 2010). The second author was also supported by the DFG research center "Mathematics for key technologies" (Matheon) in Berlin.

1 Dpto. de Matemática Aplicada y Ciencias de la Computación, E.T.S.I. Industriales y de Telecomunicación, Universidad de Cantabria, 39005 Santander, Spain. eduardo.casas@unican.es

${ }^{2}$ Institut für Mathematik, Technische Universität Berlin, 10623 Berlin, Germany. troeltzsch@math.tu-berlin.de
} 
error estimates for the discretization of equation (1.1) and associated adjoint equation. The regularity of the solutions to these equations, which is required for this analysis, is obtained from the first order necessary optimality conditions. These optimality conditions were proved in [5] and are included here for convenience.

Although the equation (1.1) is not of monotone type, it has a unique solution. This can be proved by a comparison principle. However, this technique cannot be applied to the discretized equation, where the uniqueness of the solution is an open problem. Nevertheless, we are able to derive error estimates in a local framework.

Since $(\mathrm{P})$ is not a convex problem, we have to deal with local minima. We show that every strict local minimum of $(\mathrm{P})$ can be strongly approximated by local minima of the discrete control problems.

The convergence analysis of discretized control problems associated with nonlinear elliptic equations was already studied in $[1,4]$. In both cases, the equations were semilinear. As far as we know, the specific difficulties arising from the quasilinear and non-monotone character of equation (1.1) were not yet addressed in the literature. Let us explain these difficulties as well as our contributions in this field. A first step to discretize the control problem is the approximation of the state equation (1.1), typically by using finite elements. By an application of the Brouwer fix-point theorem it is easy to deduce the existence of a solution for the discrete equation. However, in general, the uniqueness is unknown due to the non-monotone character of the equation. There are just a few uniqueness results in the case of sufficiently small data $u$, Brenner and Scott [2], pp. 188191, or when the discretization parameter $h$ is large enough, Hlaváček [9] and Hlaváček et al. [10]. Moreover these papers assume the coefficient $a(x, y)$ of the quasilinear equation to be bounded on $\Omega \times \mathbb{R}$. In this case, we are able to prove the uniqueness of the solution of the discrete equation for every $h$ small enough for any $u \in L^{p}(\Omega)$ when $p>n$; see Corollary 3.3 .

When $a(x, y)$ is not bounded, then a significant difficulty arises in formulating the discrete control problem, because the control-to-state mapping is possibly multivalued. This forces us to carry out a local analysis around a solution $\bar{u}$ of control problem $(\mathrm{P})$. We are able to prove that, around an $L^{p}(\Omega)$-ball centered at $\bar{u}$, a unique solution of the discrete state equation exists in a certain $W^{1, p}(\Omega)$-ball centered at the optimal state $\bar{y}$. To show this result, we have to derive $L^{p}$ error estimates for a finite element approximation of the state equation. To our best knowledge, these estimates are not known for our non-monotone quasilinear equation. There are some previous estimates proved by Douglas and Dupont [7] and Liu et al. [11] in the spaces $L^{2}(\Omega)$ and $H^{1}(\Omega)$, but these spaces are not suitable for our goals. Furthermore, all these papers also require the coefficient $a(x, y)$ to be bounded in $\Omega \times \mathbb{R}$.

In view of this, we are able to deal with this class of quasilinear equations under weaker assumptions and we derive more general $L^{p}$ estimates. This is not obvious due to the non-monotone character of the equation; at least it cannot be done by classical arguments. Moreover, also the uniqueness of the solution of the discrete equation for bounded coefficients $a(x, y)$ is a new contribution of this paper.

The numerical analysis of the control problem $(\mathrm{P})$ also requires the approximation of the adjoint state equation, which is linear but non-monotone. This equation has been studied by the authors in [5], where the uniqueness and regularity of the solution was investigated. Here we prove that the discrete adjoint state equation has also a unique solution in spite of its non-monotone character; see Theorem 4.1. We also derive associated $L^{p}$ error estimates in Theorem 4.5.

\section{Assumptions AND PRELIMinary RESUlts}

The following hypotheses will be assumed in the whole paper.

(H1) $\Omega$ is an open, convex and bounded subset of $\mathbb{R}^{n}, n=2$ or 3 , with boundary $\Gamma$ of class $C^{1,1}$. We fix real numbers $\alpha<\beta$ and introduce the admissible set

$$
\mathcal{U}_{a d}=\left\{u \in L^{\infty}(\Omega): \alpha \leq u(x) \leq \beta \text { for a.e. } x \in \Omega\right\}
$$


(H2) The function $a: \bar{\Omega} \times \mathbb{R} \longrightarrow \mathbb{R}$ is of class $C^{1}$ with respect to the second component and, for any $M>0$, there exists a constant $C_{a, M}>0$ such that, for all $x_{i} \in \bar{\Omega}$ and $\left|y_{i}\right| \leq M, i=1,2$, it holds

$$
\left|\frac{\partial^{j} a}{\partial y^{j}}\left(x_{2}, y_{2}\right)-\frac{\partial^{j} a}{\partial y^{j}}\left(x_{1}, y_{1}\right)\right| \leq C_{a, M}\left(\left|x_{2}-x_{1}\right|+\left|y_{2}-y_{1}\right|\right), j=0,1 .
$$

Moreover, we assume

$$
\exists a_{0}>0 \text { such that } a(x, y) \geq a_{0} \quad \forall x \in \Omega, \forall y \in \mathbb{R} .
$$

(H3) The function $f: \Omega \times \mathbb{R} \longrightarrow \mathbb{R}$ is measurable with respect to the first variable, it is of class $C^{1}$ with respect to the second, and the following properties hold:

$$
\begin{aligned}
& \exists \bar{p}>n \text { such that } f(\cdot, 0) \in L^{\bar{p}}(\Omega) \\
& \frac{\partial f}{\partial y}(x, y) \geq 0 \text { for a.e. } x \in \Omega, \forall y \in \mathbb{R} \text { and } \frac{\partial f}{\partial y}(\cdot, 0) \in L^{\infty}(\Omega) \\
& \forall M>0 \exists C_{f, M}>0 \text { such that }\left|\frac{\partial f}{\partial y}\left(x, y_{1}\right)-\frac{\partial f}{\partial y}\left(x, y_{2}\right)\right| \leq C_{f, M}\left|y_{1}-y_{2}\right|
\end{aligned}
$$

for almost all $x \in \Omega$ and all $\left|y_{1}\right|,\left|y_{2}\right| \leq M$.

(H4) The function $L: \Omega \times \mathbb{R} \times \mathbb{R} \longrightarrow \mathbb{R}$ is measurable with respect to the first variable, of class $C^{1}$ with respect to the others, and twice differentiable with respect to $u$. Moreover

$$
\begin{aligned}
& L(\cdot, 0,0) \in L^{1}(\Omega), \frac{\partial^{2} L}{\partial u^{2}}(x, y, u) \geq \Lambda>0 \text { a.e. in } \Omega \text { and for all } y, u \in \mathbb{R} \\
& \forall M>0 \exists C_{L, M}>0 \text { and } \psi_{L, M} \in L^{\bar{p}}(\Omega) \text { such that } \\
& \left|\frac{\partial L}{\partial u}(x, y, u)\right| \leq C_{L, M} \\
& \left|\frac{\partial L}{\partial y}(x, y, u)\right| \leq \psi_{L, M}(x) \\
& \left|\frac{\partial L}{\partial u}\left(x_{2}, y, u\right)-\frac{\partial L}{\partial u}\left(x_{1}, y, u\right)\right| \leq C_{L, M}\left|x_{2}-x_{1}\right|
\end{aligned}
$$

for all $x, x_{i} \in \Omega, y \in \mathbb{R}$, where $\bar{p}>n$ is as in Hypothesis (H3).

These assumptions look somewhat technical, but we aimed to include the associated most general case. The following more special example falls into this class:

$$
\text { (E) }\left\{\begin{array}{l}
\min \int_{\Omega}\left(y_{u}(x)-y_{d}(x)\right)^{2}+\nu u(x)^{2} \mathrm{~d} x, \\
\alpha \leq u(x) \leq \beta \text { for } \text { a.e. } x \in \Omega,
\end{array}\right.
$$

where $y_{u}$ is the solution of the state equation

$$
\left\{\begin{aligned}
-\operatorname{div}[a(x, y) \nabla y]+\exp (y) & =u(x) & & \text { in } \Omega \\
y & =0 & & \text { on } \Gamma
\end{aligned}\right.
$$

with $y_{d} \in L^{\infty}(\Omega), \nu, \lambda>0$, and $a \in C^{1}(\bar{\Omega} \times \mathbb{R})$. For instance, $a(x, y)=\phi_{0}(x)+y^{2}$ with a Lipschitz function $\phi_{0}(x) \geq \alpha_{0}>0$ meets our assumptions.

For the state equation, we have the following result. 
Theorem 2.1. For every $u \in L^{p}(\Omega)$, with $2 \leq p \leq \bar{p}$, the state equation (1.1) has a unique solution $y_{u} \in$ $W^{2, p}(\Omega) \cap W_{0}^{1, p}(\Omega)$, which depends continuously on $u$. Moreover, for all bounded sets $\mathcal{K} \subset L^{p}(\Omega)$, there exists $C_{\mathcal{K}}>0$ such that

$$
\left\|y_{u}\right\|_{W^{2, p}(\Omega)} \leq C_{\mathcal{K}} \quad \forall u \in \mathcal{K} .
$$

Notice that we cannot expect a higher regularity of $y_{u}$ for $p>\bar{p}$, since the regularity of $f$ according to (2.3) limits the regularity of $u+f$.

For the proof of this theorem, we refer the reader to Casas and Tröltzsch [5]. Moreover, the solution $y_{u}$ depends continuously of $u$. In particular, there exists a constant $C_{\alpha, \beta}>0$ such that

$$
\left\|y_{u}\right\|_{W^{2, \bar{p}}(\Omega)} \leq C_{\alpha, \beta} \quad \forall u \in \mathcal{U}_{a d} .
$$

On the other hand, $\bar{p}>n$ implies that $W^{2, \bar{p}}(\Omega) \subset C^{1}(\bar{\Omega})$, hence all the feasible states of problem (P) are $C^{1}$-functions in $\bar{\Omega}$.

We also need the following result of [5] on the differentiability of the control-to-state mapping:

Theorem 2.2. The mapping $G: L^{\infty}(\Omega) \longrightarrow W^{2, \bar{p}}(\Omega)$, defined by $G(u)=y_{u}$, is of class $C^{1}$. For any $v \in$ $L^{\infty}(\Omega)$, the function $z_{v}=G^{\prime}(u) v$ is the unique solution in $W^{2, \bar{p}}(\Omega) \cap W_{0}^{1, \bar{p}}(\Omega)$ of the equation

$$
\left\{\begin{aligned}
-\operatorname{div}\left[a\left(x, y_{u}\right) \nabla z+\frac{\partial a}{\partial y}\left(x, y_{u}\right) z \nabla y_{u}\right]+\frac{\partial f}{\partial y}\left(x, y_{u}\right) z & =v \text { in } \Omega \\
z & =0 \text { on } \Gamma .
\end{aligned}\right.
$$

The proof of this theorem relies crucially on the fact that the linearized equation (2.12) has a unique solution in $H_{0}^{1}(\Omega)$. Moreover, this solution has $W^{2, \bar{p}}(\Omega)$-regularity. Although the equation (2.12) is not monotone, the authors were able to prove the well posedness of the equation in [5]. In fact, for any $v \in W^{-1, p}(\Omega)$, the boundary value problem

$$
\left\{\begin{aligned}
-\operatorname{div}\left[a\left(x, y_{1}\right) \nabla z+\frac{\partial a}{\partial y}\left(x, y_{2}\right) z \nabla y\right]+\frac{\partial f}{\partial y}\left(x, y_{3}\right) z & =v \text { in } \Omega \\
z & =0 \text { on } \Gamma
\end{aligned}\right.
$$

has a unique solution $z \in W_{0}^{1, p}(\Omega)$ provided that $n<p \leq \bar{p}, y_{1} \in C(\bar{\Omega}), y_{2}, y_{3} \in L^{\infty}(\Omega)$, and $y \in W^{1, p}(\Omega)$.

Remark 2.3. The mapping $G$ introduced in Theorem 2.2 can be extended to a $C^{1}$-mapping $G: L^{2}(\Omega) \longrightarrow$ $H^{2}(\Omega)$. In particular, equation (2.12) defines an isomorphism $v \mapsto z_{v}$ between $L^{2}(\Omega)$ and $H_{0}^{1}(\Omega) \cap H^{2}(\Omega)$.

By the previous theorem and our assumptions on the given functions of the control problem, we obtained the following result [5]:

Theorem 2.4. The functional $J: L^{\infty}(\Omega) \rightarrow \mathbb{R}$ is of class $C^{1}$ and for every $u, v \in L^{\infty}(\Omega)$ we have

$$
J^{\prime}(u) v=\int_{\Omega}\left(\frac{\partial L}{\partial u}\left(x, y_{u}, u\right)+\varphi_{u}\right) v \mathrm{~d} x
$$

where $\varphi_{u} \in W_{0}^{1, \bar{p}}(\Omega) \cap W^{2, \bar{p}}(\Omega)$ is the unique solution of the problem

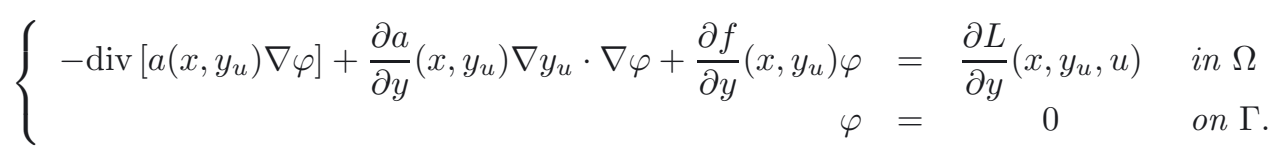

Now, we already have the main tools to study the control problem (P). First of all, the reader can easily check that $(\mathrm{P})$ has at least one global solution. The proof follows by standard arguments. In the rest of this section we will formulate the first order optimality conditions corresponding to local minima of $(\mathrm{P})$. They will lead us to a regularity result for local minima. 
We say that $\bar{u}$ is a local minimum of $(\mathrm{P})$ if there exists an open ball $B_{\varepsilon}(\bar{u})$ in $L^{\infty}(\Omega)$ such that

$$
J(\bar{u}) \leq J(u) \quad \forall u \in \mathcal{U}_{a d} \cap B_{\varepsilon}(\bar{u})
$$

Also the next two theorems were proven in [5]:

Theorem 2.5. If $\bar{u}$ is a local solution of $(\mathrm{P})$ with associated state $\bar{y}=y_{\bar{u}}$, then there exists an adjoint state function $\bar{\varphi}$ in $W^{2, \bar{p}}(\Omega)$ such that the following optimality system is satisfied:

$$
\begin{aligned}
& \left\{\begin{array}{rlcc}
-\operatorname{div}[a(x, \bar{y}(x)) \nabla \bar{y}(x)]+f(x, \bar{y}(x)) & =\bar{u}(x) & \text { in } \Omega \\
\bar{y}(x) & =0 & \text { on } \Gamma,
\end{array}\right.
\end{aligned}
$$

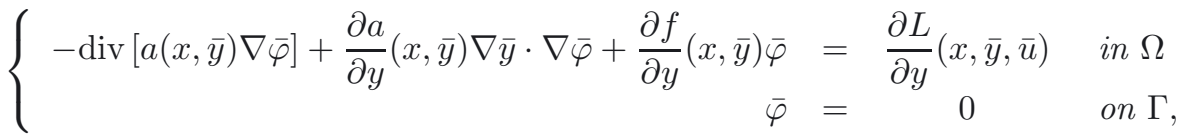

$$
\begin{aligned}
& \int_{\Omega}\left(\frac{\partial L}{\partial u}(x, \bar{y}(x), \bar{u}(x))+\bar{\varphi}(x)\right)(u(x)-\bar{u}(x)) \mathrm{d} x \geq 0 \quad \forall u \in \mathcal{U}_{a d} .
\end{aligned}
$$

Remark 2.6. Given $y \in W^{1, p}(\Omega)$, let us consider the partial differential operator

$$
A(y) z=-\operatorname{div}\left[a(x, y) \nabla z+\frac{\partial a}{\partial y}(x, y) z \nabla y\right]+\frac{\partial f}{\partial y}(x, y) z
$$

The formally adjoint operator is

$$
A(y)^{*} \varphi=-\operatorname{div}[a(x, y) \nabla \varphi]+\frac{\partial a}{\partial y}(x, y) \nabla y \nabla \varphi+\frac{\partial f}{\partial y}(x, y) \varphi .
$$

In [5], Theorem 3.2, it was proved that $A(y)$ and $A(y)^{*}$ are isomorphisms from $W_{0}^{1, p}(\Omega)$ to $W^{-1, p}(\Omega)$ for $2 \leq p \leq \bar{p}$.

From (2.18) we get in a standard way

$$
\bar{u}(x)=\left\{\begin{array}{ll}
\alpha & \text { if } \bar{d}(x)>0 \\
\beta & \text { if } \bar{d}(x)<0
\end{array} \quad \text { and } \bar{d}(x)= \begin{cases}\geq 0 & \text { if } \bar{u}(x)=\alpha \\
\leq 0 & \text { if } \bar{u}(x)=\beta \\
=0 & \text { if } \alpha<\bar{u}(x)<\beta\end{cases}\right.
$$

for almost all $x \in \Omega$, where

$$
\bar{d}(x)=\frac{\partial L}{\partial u}(x, \bar{y}(x), \bar{u}(x))+\bar{\varphi}(x) .
$$

Furthermore, (2.18) allows us to deduce some higher regularity of the local minimum $\bar{u}$.

Theorem 2.7. If $\bar{u}$ is a local minimum of $(\mathrm{P})$, then, for every $x \in \bar{\Omega}$, the equation

$$
\frac{\partial L}{\partial u}(x, \bar{y}(x), t)+\bar{\varphi}(x)=0
$$

has a unique solution $\bar{t}=\bar{s}(x)$. The function $\bar{s}: \bar{\Omega} \rightarrow \mathbb{R}$ is Lipschitz and $\bar{u}$ is related to $\bar{s}$ by the formula

$$
\bar{u}(x)=\operatorname{Proj}_{[\alpha, \beta]}(\bar{s}(x))=\max \{\min \{\beta, \bar{s}(x)\}, \alpha\} .
$$

Consequently, $\bar{u}$ is Lipschitz in $\bar{\Omega}$, too. 


\section{Numerical ANALYSis OF THE STATE EQUATION}

The goal of this section is to study the approximation of the state equation (1.1) by finite elements and to derive some associated error estimates. To this aim, we consider a family of triangulations $\left\{\mathcal{T}_{h}\right\}_{h>0}$ of $\bar{\Omega}$, defined in the standard way, e.g. in [2]. In particular, this definition excludes the so-called hanging nodes. Moreover, this triangulation is supposed to be regular and to satisfy an inverse assumption; see (i) below.

With each element $T \in \mathcal{T}_{h}$, we associate two parameters $\rho(T)$ and $\sigma(T)$, where $\rho(T)$ denotes the diameter of the set $T$ and $\sigma(T)$ is the diameter of the largest ball contained in $T$. Define the size of the mesh by $h=\max _{T \in \mathcal{T}_{h}} \rho(T)$. We suppose that the following regularity assumptions are satisfied.

(i) There exist two positive constants $\rho$ and $\sigma$ such that

$$
\frac{\rho(T)}{\sigma(T)} \leq \sigma, \quad \frac{h}{\rho(T)} \leq \rho
$$

hold for all $T \in \mathcal{T}_{h}$ and all $h>0$.

(ii) Define $\bar{\Omega}_{h}=\cup_{T \in \mathcal{T}_{h}} T$, and let $\Omega_{h}$ and $\Gamma_{h}$ denote its interior and its boundary, respectively. We assume that $\bar{\Omega}_{h}$ is convex and that the vertices of $\mathcal{T}_{h}$ placed on the boundary $\Gamma_{h}$ are points of $\Gamma$. From [13], estimate (5.2.19), we know that

$$
\left|\Omega \backslash \Omega_{h}\right| \leq C h^{2}
$$

We will use piecewise linear approximations for the states, thus we set

$$
Y_{h}=\left\{y_{h} \in C(\bar{\Omega}) \mid y_{h \mid T} \in \mathcal{P}_{1} \text {, for all } T \in \mathcal{T}_{h}, \text { and } y_{h}=0 \text { on } \bar{\Omega} \backslash \Omega_{h}\right\}
$$

where $\mathcal{P}_{1}$ is the space of polynomials of degree less or equal than 1.

The discrete version of equation (1.1) is defined as follows:

$$
\left\{\begin{array}{l}
\text { Find } y_{h} \in Y_{h} \text { such that, for all } z_{h} \in Y_{h}, \\
\int_{\Omega_{h}}\left[a\left(x, y_{h}(x)\right) \nabla y_{h}(x) \nabla z_{h}(x)+f\left(x, y_{h}(x)\right) z_{h}(x)\right] \mathrm{d} x=\int_{\Omega_{h}} u(x) z_{h}(x) \mathrm{d} x .
\end{array}\right.
$$

By applying the Brouwer fixed point theorem, using (2.2)-(2.4) and taking into account that $Y_{h} \subset C(\bar{\Omega})$, it is easy to deduce the existence of at least one solution of (3.2). As far as we know, the uniqueness was an open question until now. There are some uniqueness results for the restrictive cases where $u$ is sufficiently small or $h$ is large enough; see [2], pp. 188-191, and [9,10]. In the previous references, the functions $a$ and $f$ are assumed to be bounded in $\Omega \times \mathbb{R}$.

We are are able to prove a more general uniqueness theorem: if $a$ and $f$ are bounded, then there exists an $h_{0}>0$ such that (3.2) has a unique solution for every $h<h_{0}$ and any $u \in \mathcal{U}_{a d}$, where $h_{0}$ is independent of $u$. We also derive some error estimates. If we do not assume the boundedness of the functions $a$ and $f$, then we will prove that the solution of (1.1) can be approximated by solutions of (3.2) and we derive estimates for these approximations. The question of the existence of other solutions $y_{h}$ of (3.2), which are not close to the solution $y$ of (1.1), remains open. If such solutions $y_{h}$ exist, then $\left\|y_{h}\right\|_{L^{\infty}(\Omega)} \rightarrow \infty$ when $h \rightarrow 0$; see Corollary 3.3.

Theorem 3.1. Let $\mathcal{K} \subset L^{p}(\Omega)$, with $2 \leq p \leq \bar{p}$, be a bounded subset. There exist two constants $h_{0}>0$ and $C_{\mathcal{K}}>0$ such that, for any $u \in \mathcal{K}$ and $h<h_{0}$, equation (3.2) has at least one solution $y_{h}(u)$ that obeys

$$
\begin{aligned}
& \left\|y_{u}-y_{h}(u)\right\|_{L^{2}\left(\Omega_{h}\right)}+h\left\|y_{u}-y_{h}(u)\right\|_{H^{1}\left(\Omega_{h}\right)} \leq C_{\mathcal{K}} h^{2} \\
& \left\|y_{u}-y_{h}(u)\right\|_{W^{1, p}\left(\Omega_{h}\right)} \leq \begin{cases}C_{\mathcal{K}} h^{\frac{2}{p}} & \text { if } n=2 \\
C_{\mathcal{K}} h^{\frac{6-p}{2 p}} & \text { if } n=3 \text { and } p<6,\end{cases}
\end{aligned}
$$


where $y_{u}$ is the solution of (1.1). If a and $f$ are bounded in $\Omega \times \mathbb{R}$, then (3.3) and (3.4) hold for all the solutions of (3.2). Finally, if $y_{h}$ is a solution of (3.2) for every $h>0$ and the family $\left\{y_{h}\right\}_{h>0}$ is bounded in $L^{\infty}(\Omega)$, then the inequalities (3.3) and (3.4) hold with $y_{h}$ substituted for $y_{h}(u)$.

Proof. (i) Proof of (3.3). Let us take

$$
M=1+\sup \left\{\left\|y_{v}\right\|_{C(\bar{\Omega})}: v \in \mathcal{K}\right\}
$$

According to Theorem 2.1, it holds that $M<\infty$. Now we consider a nondecreasing cut-off function $\phi_{M}: \mathbb{R} \longrightarrow \mathbb{R}$ of class $C^{\infty}$ such that

$$
\phi_{M}(t)=\left\{\begin{array}{cl}
t & \text { if }|t| \leq M \\
M+1 & \text { if } t \geq M+1 \\
-M-1 & \text { if } t \leq-M-1
\end{array}\right.
$$

and we set $a_{M}(x, y)=a\left(x, \phi_{M}(y)\right)$ and $f_{M}(x, y)=f\left(x, \phi_{M}(y)\right)$. Then $a_{M}$ and $f_{M}$ are functions of class $C^{1}$ with respect to the second variable and $\partial_{y}^{j} a_{M}(x, y)$ and $\partial_{y}^{j} f_{M}(x, y)$ are bounded in $\bar{\Omega} \times \mathbb{R}$ for $j=0,1$. Let us consider the equation

$$
\left\{\begin{array}{rccc}
-\operatorname{div}\left[a_{M}(x, y(x)) \nabla y(x)\right]+f_{M}(x, y(x)) & = & u(x) & \text { in } \Omega \\
y(x) & = & 0 & \text { on } \Gamma
\end{array}\right.
$$

and its discrete version

$$
\left\{\begin{array}{l}
\text { Find } y_{h} \in Y_{h} \text { such that } \forall z_{h} \in Y_{h} \\
\int_{\Omega_{h}}\left[a_{M}\left(x, y_{h}(x)\right) \nabla y_{h} \nabla z_{h}+f_{M}\left(x, y_{h}(x)\right) z_{h}\right] \mathrm{d} x=\int_{\Omega_{h}} u z_{h} \mathrm{~d} x .
\end{array}\right.
$$

From (3.5) we get that $a_{M}\left(x, y_{u}(x)\right)=a\left(x, y_{u}(x)\right)$ and $f_{M}\left(x, y_{u}(x)\right)=f\left(x, y_{u}(x)\right)$ for every $x \in \Omega$ and all $u \in \mathcal{K}$. Therefore (3.6) has the same solution than (1.1) for any $u \in \mathcal{K}$. Let us denote by $y_{h}^{M}(u)$ an arbitrary but fixed solution of (3.2). Now we can apply the error estimates obtained in [11] for the quasilinear problems (3.6) and (3.7) and deduce the existence of a constant $C_{1}>0$ depending on $\left\|y_{u}\right\|_{H^{2}(\Omega)}$ such that

$$
\left\|y_{u}-y_{h}^{M}(u)\right\|_{L^{2}\left(\Omega_{h}\right)}+h\left\|y_{u}-y_{h}^{M}(u)\right\|_{H^{1}\left(\Omega_{h}\right)} \leq C_{M} h^{2}
$$

where $C_{M}$ is independent on $h$ and $u \in \mathcal{K}$.

The equation considered in [11] does not contain the semilinear term $f_{M}(x, y)$, but it can be treated similarly as $a_{M}$, even in a easier way.

Now denote by $\Pi_{h}: C(\bar{\Omega}) \longrightarrow Y_{h}$ the interpolation operator. It is known that, for all $z \in H^{2}(\Omega)$,

$$
\left\{\begin{array}{l}
\left\|z-\Pi_{h} z\right\|_{L^{2}\left(\Omega_{h}\right)}+h\left\|z-\Pi_{h} z\right\|_{H^{1}\left(\Omega_{h}\right)} \leq C_{1} h^{2}\|z\|_{H^{2}(\Omega)}, \\
\left\|z-\Pi_{h} z\right\|_{L^{\infty}\left(\Omega_{h}\right)} \leq C_{1} h^{2-\frac{n}{2}}\|z\|_{H^{2}(\Omega)}
\end{array}\right.
$$

see, for instance, Ciarlet [6]. The same book also contains the inverse inequality

$$
\left\|z_{h}\right\|_{L^{\infty}\left(\Omega_{h}\right)} \leq C_{2} h^{-\frac{n}{2}}\left\|z_{h}\right\|_{L^{2}\left(\Omega_{h}\right)} \quad \forall z_{h} \in Y_{h}
$$


Using (3.3), (3.9) and (3.10) we get

$$
\begin{aligned}
\left\|y_{u}-y_{h}^{M}(u)\right\|_{L^{\infty}\left(\Omega_{h}\right)} & \leq\left\|y_{u}-\Pi_{h} y_{u}\right\|_{L^{\infty}\left(\Omega_{h}\right)}+\left\|\Pi_{h} y_{u}-y_{h}^{M}(u)\right\|_{L^{\infty}\left(\Omega_{h}\right)} \\
& \leq C_{1} h^{2-\frac{n}{2}}\left\|y_{u}\right\|_{H^{2}(\Omega)}+C_{2} h^{-\frac{n}{2}}\left\|\Pi_{h} y_{u}-y_{h}^{M}(u)\right\|_{L^{2}\left(\Omega_{h}\right)} \\
& \leq C_{1} h^{2-\frac{n}{2}}\left\|y_{u}\right\|_{H^{2}(\Omega)}+C_{2} h^{-\frac{n}{2}}\left(\left\|\Pi_{h} y_{u}-y_{u}\right\|_{L^{2}\left(\Omega_{h}\right)}+\left\|y_{u}-y_{h}^{M}(u)\right\|_{L^{2}\left(\Omega_{h}\right)}\right) \\
& \leq C_{1} h^{2-\frac{n}{2}}\left\|y_{u}\right\|_{H^{2}(\Omega)}+C_{2} h^{2-\frac{n}{2}}\left(C_{1}+C_{M}\right)\left\|y_{u}\right\|_{H^{2}(\Omega)} .
\end{aligned}
$$

This inequality, along with (2.11), leads to

$$
\left\|y_{u}-y_{h}^{M}(u)\right\|_{L^{\infty}\left(\Omega_{h}\right)} \leq \begin{cases}\hat{C}_{M} h & \text { if } n=2 \\ \hat{C}_{M} h^{\frac{1}{2}} & \text { if } n=3\end{cases}
$$

Therefore there exists $h_{0}>0$ such that

$$
\left\|y_{u}-y_{h}^{M}(u)\right\|_{L^{\infty}\left(\Omega_{h}\right)}<1 \quad \forall h<h_{0} .
$$

This inequality implies that

$$
\left\|y_{h}^{M}(u)\right\|_{L^{\infty}(\Omega)}=\left\|y_{h}^{M}(u)\right\|_{L^{\infty}\left(\Omega_{h}\right)} \leq\left\|y_{u}-y_{h}^{M}(u)\right\|_{L^{\infty}\left(\Omega_{h}\right)}+\left\|y_{u}\right\|_{C(\bar{\Omega})}<1+\left\|y_{u}\right\|_{C(\bar{\Omega})} \leq M \quad \forall h<h_{0} .
$$

Therefore the following identities hold for $h<h_{0}$

$$
a_{M}\left(x, y_{h}^{M}(u)(x)\right)=a\left(x, y_{h}^{M}(u)(x)\right) \text { and } f_{M}\left(x, y_{h}^{M}(u)(x)\right)=f\left(x, y_{h}^{M}(u)(x)\right),
$$

thus $y_{h}(u)=y_{h}^{M}(u)$ is a solution of (3.2) and (3.3) follows from (3.8).

(ii) Proof of (3.4). By estimates for the interpolation error and inverse inequalities of [6], we get

$$
\left\|z-\Pi_{h} z\right\|_{W^{1, p}\left(\Omega_{h}\right)} \leq C_{1} h\|z\|_{W^{2, p}(\Omega)} \quad \forall z \in W^{2, p}(\Omega) \quad \text { and } \quad\left\|z_{h}\right\|_{W^{1, p}\left(\Omega_{h}\right)} \leq \frac{C_{2}}{h^{\frac{n(p-2)}{2 p}}}\left\|z_{h}\right\|_{H^{1}\left(\Omega_{h}\right)} \quad \forall z_{h} \in Y_{h} .
$$

Using (3.3) and the previous inequalities it follows that

$$
\begin{aligned}
\left\|y_{u}-y_{h}(u)\right\|_{W^{1, p}\left(\Omega_{h}\right)} & \leq\left\|y_{u}-\Pi_{h} y_{u}\right\|_{W^{1, p}\left(\Omega_{h}\right)}+\left\|\Pi_{h} y_{u}-y_{h}(u)\right\|_{W^{1, p}\left(\Omega_{h}\right)} \\
& \leq C_{1} h\left\|y_{u}\right\|_{W^{2, p}(\Omega)}+\frac{C_{2}}{h^{\frac{n(p-2)}{2 p}}}\left\|\Pi_{h} y_{u}-y_{h}(u)\right\|_{H^{1}\left(\Omega_{h}\right)} \\
& \leq C_{1} h\left\|y_{u}\right\|_{W^{2, p}(\Omega)}+\frac{C_{2}}{h^{\frac{n(p-2)}{2 p}}}\left(\left\|\Pi_{h} y_{u}-y_{u}\right\|_{H^{1}\left(\Omega_{h}\right)}+\left\|y_{u}-y_{h}(u)\right\|_{H^{1}\left(\Omega_{h}\right)}\right) \\
& \leq C_{1} h\left\|y_{u}\right\|_{W^{2, p}(\Omega)}+C_{2} h^{1+\frac{n(2-p)}{2 p}}\left(C_{1}+C_{\mathcal{K}}\right)\left\|y_{u}\right\|_{H^{2}(\Omega)} .
\end{aligned}
$$

For $n=2$, the number $1+n(2-p) / 2 p$ above is equal to $2 / p$, hence the upper exponent in (3.4) is found. If $n=3$, then we obtain the value $(6-p) / 2 p$, which is positive for $p<6$. Therefore, the last inequality, along with the estimate of Theorem 2.1, yields (3.4). 
If the functions $a$ and $f$ are bounded, then we do not need the cut off function $\phi_{M}$ and we can get directly the inequalities (3.3) and (3.4) for any solution of (3.2). Finally, if $\left\{y_{h}\right\}_{h>0}$ is a family of solution of (3.2) bounded in $L^{\infty}(\Omega)$, then we can define $a_{M}$ and $f_{M}$ as above, with

$$
M=\sup _{h>0}\left\|y_{h}\right\|_{L^{\infty}(\Omega)}+\left\|y_{u}\right\|_{L^{\infty}(\Omega)}+1
$$

Then we can apply the results of [11] to the equations (3.6) and (3.7) and deduce that $y_{h}$ satisfies (3.3) and consequently (3.4) too.

In the rest of the section, we fix $\bar{u} \in L^{p}(\Omega)$ and denote by $\bar{y}$ the solution of (1.1) associated to $\bar{u}$, precisely $\bar{y}=y_{\bar{u}}$. From Theorem 2.1 we know that $\bar{y} \in W^{2, p}(\Omega)$. We are going to prove the following theorem on existence and uniqueness and on an $L^{p}$-error estimate:

Theorem 3.2. Suppose that $n<p \leq \bar{p}$. Then there exist $h_{0}>0, \rho_{\bar{u}}>0$ and $\rho_{\bar{y}}>0$ such that, for any $h<h_{0}$ and any $u \in \bar{B}_{\rho_{\bar{u}}}(\bar{u}) \subset L^{p}(\Omega)$, equation (3.2) has in the closed ball $\bar{B}_{\rho_{\bar{y}}}(\bar{y}) \subset W_{0}^{1, p}(\Omega)$ a unique solution $y_{h}(u)$. Moreover, there holds the estimate

$$
\left\|y_{u}-y_{h}(u)\right\|_{L^{p}\left(\Omega_{h}\right)}+h\left\|y_{u}-y_{h}(u)\right\|_{W^{1, p}\left(\Omega_{h}\right)} \leq C(\bar{u}) h^{2} .
$$

The proof of Theorem 3.2 is worked out in the next subsections.

As a consequence of Theorems 3.1 and 3.2, we get the following result.

Corollary 3.3. Assume that $n<p \leq \bar{p}$ and $\bar{u} \in L^{p}(\Omega)$, then the following statements hold.

(1) If the functions $a$ and $f$ are bounded in $\Omega \times \mathbb{R}$, then there exists $h_{0}>0$ such that (3.2) has a unique solution for every $h \leq h_{0}$.

(2) Suppose that, as in Theorem 3.2, (3.2) has in the ball $B_{\rho_{y_{u}}}\left(y_{u}\right) \subset W_{0}^{1, p}(\Omega)$ for every $h<h_{0}$ a unique solution. If for any $h<h_{0}$ there exists another solution $y_{h}$ of (3.2) outside the ball $B_{\rho_{y_{u}}}\left(y_{u}\right)$, then

$$
\lim _{h \rightarrow 0}\left\|y_{h}\right\|_{L^{\infty}(\Omega)}=+\infty
$$

Proof. Let us show (1) under the simplifying assumption that the estimate (3.8) is valid that was used in the proof of Theorem 3.1. This estimate was shown in [10] under the fairly strong assumption that, in addition to $a$, also the first- and second-order derivatives of $a$ with respect to $y$ are bounded. This simplification is not necessary as the proof of Theorem 4.1 in the forthcoming paper [3] shows. In [3], a statement analogous to (1) is proven for bounded $a$ in the case of boundary control. The adaptation of this proof to our case of distributed control is straightforward.

If $n=3$ we can assume without loss of generality that that $p<6$. Indeed, if we prove the uniqueness of solutions for data $u \in L^{p}(\Omega)$, with $p<6$, then the result is obviously true for $p \geq 6$. From Theorem 3.2 we deduce the existence of $h_{1}>0$ and $\rho_{y_{u}}>0$ such that (3.2) has a unique solution in $B_{\rho_{y_{u}}}\left(y_{u}\right) \subset W_{0}^{1, p}(\Omega)$ for every $h<h_{1}$. Let now $y_{h}(u)$ be any solution of (3.2). Then, Theorem 3.1 yields the existence of $h_{2}>0$ such that

$$
\left\|y_{u}-y_{h}(u)\right\|_{W_{0}^{1, p}(\Omega)}<\rho_{y_{u}} \forall h<h_{2} .
$$

To check this we notice first that $y_{u}=y_{u}-y_{h}(u)$ in $\Omega \backslash \Omega_{h}$ and $y_{u} \in W^{2, p}(\Omega) \subset C^{1}(\bar{\Omega})$ thanks to $p>n$. Then the previous inequality is a consequence of (3.1), (3.3) and (3.4)

$$
\left\|y_{u}-y_{h}(u)\right\|_{W_{0}^{1, p}(\Omega)} \leq C\left\|y_{u}\right\|_{C^{1}\left(\bar{\Omega} \backslash \Omega_{h}\right)}\left|\Omega \backslash \Omega_{h}\right|^{1 / p}+\left\|y_{u}-y_{h}(u)\right\|_{W_{0}^{1, p}\left(\Omega_{h}\right)} \leq C^{\prime}\left\{h^{\frac{2}{p}}\left\|y_{u}\right\|_{C^{1}\left(\bar{\Omega} \backslash \Omega_{h}\right)}+h^{s}\right\}
$$


where

$$
s=\left\{\begin{array}{cl}
\frac{2}{p} & \text { if } n=2, \\
\frac{6-p}{2 p} & \text { if } n=3 \text { and } p<6 .
\end{array}\right.
$$

By taking $h_{0}=\min \left\{h_{1}, h_{2}\right\}$ we get that any solution of (3.2) for $h<h_{0}$ belongs to the ball $B_{\rho_{y_{u}}}\left(y_{u}\right)$, which implies the uniqueness.

To prove (2), we proceed by contradiction. Let us assume that $\left\{y_{h}\right\}_{h<h_{0}} \subset Y_{h} \backslash B_{\rho_{y_{u}}}\left(y_{u}\right)$ is bounded in $L^{\infty}(\Omega)$. We denote by $y_{h}(u)$ the solution of (3.2) belonging to the ball $B_{\rho_{y_{u}}}\left(y_{u}\right)$. Then we define

$$
M=\max _{h<h_{0}}\left\|y_{h}\right\|_{L^{\infty}(\Omega)}+\max _{h<h_{0}}\left\|y_{h}(u)\right\|_{L^{\infty}(\Omega)}+\left\|y_{u}\right\|_{L^{\infty}(\Omega)}+1 .
$$

Taking $a_{M}$ and $f_{M}$ as in the proof of Theorem 3.1, we have that $y_{h}$ and $y_{h}(u)$ are solutions of (3.7) for every $h<h_{0}$, which contradicts (1) (notice that $a_{M}$ and $f_{M}$ are bounded).

\subsection{Existence and uniqueness of solutions of (3.2) around $\bar{u}$}

Let us consider the mapping

$$
\mathcal{F}: L^{p}(\Omega) \times W_{0}^{1, p}(\Omega) \longrightarrow W_{0}^{1, p}(\Omega), \quad \mathcal{F}(u, y)=z-y,
$$

where $z$ is the solution of the linear equation

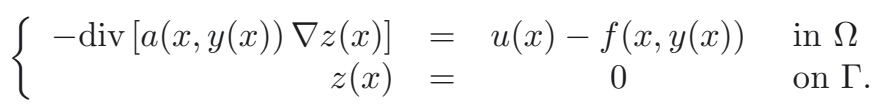

Following the steps of Theorem 2.4 of [5], we obtain $z \in W^{2, p}(\Omega)$; therefore $\mathcal{F}$ is well defined. The next proposition states some properties of this function. Let us provide first the expression for the derivative $\frac{\partial \mathcal{F}}{\partial y}(u, y)$ in $W_{0}^{1, p}(\Omega)$. It is given by

where $z_{w}$ is the solution of

$$
\frac{\partial \mathcal{F}}{\partial y}(u, y) w=z_{w}-w
$$

$$
\left\{\begin{aligned}
-\operatorname{div}\left[a(x, y) \nabla z_{w}+\frac{\partial a}{\partial y}(x, y) w \nabla z\right]+\frac{\partial f}{\partial y}(x, y) w & =0 \text { in } \Omega \\
z_{w} & =0 \text { on } \Gamma
\end{aligned}\right.
$$

and $z$ is defined by (3.13).

Proposition 3.4. The following properties hold.

(1) $\mathcal{F}(u, y)=0$ if and only if $y=y_{u}$.

(2) $\mathcal{F}$ is of class $C^{1}$.

(3) The linear mapping $\frac{\partial \mathcal{F}}{\partial y}(u, y): W_{0}^{1, p}(\Omega) \longrightarrow W_{0}^{1, p}(\Omega)$, is an isomorphism.

Proof. The statements (1) and (2) are obvious. The formula for the partial derivative of $\mathcal{F}$ defined above is also evident. Let us prove that $\frac{\partial \mathcal{F}}{\partial y}(u, y)$ is an isomorphism in $W_{0}^{1, p}(\Omega)$. To show the injectivity, we assume that $w \in W_{0}^{1, p}(\Omega)$ and

$$
z_{w}-w=\frac{\partial \mathcal{F}}{\partial y}(u, y) w=0
$$


Then $w$ satisfies the equation

$$
\left\{\begin{aligned}
-\operatorname{div}\left[a(x, y) \nabla w+\frac{\partial a}{\partial y}(x, y) w \nabla z\right]+\frac{\partial f}{\partial y}(x, y) w & =0 \text { in } \Omega \\
w & =0 \text { on } \Gamma .
\end{aligned}\right.
$$

From the uniqueness of the solution of the linear equation $(2.13)$ we deduce that $w=0$.

Now, we verify the surjectivity. Given $\phi \in W_{0}^{1, p}(\Omega)$, to solve $\frac{\partial \mathcal{F}}{\partial y}(u, y) w=\phi$, we define $w$ as the solution of

$$
\left\{\begin{array}{rlrl}
-\operatorname{div}\left[a(x, y) \nabla w+\frac{\partial a}{\partial y}(x, y) w \nabla z\right]+\frac{\partial f}{\partial y}(x, y) w & =\operatorname{div}[a(x, y) \nabla \phi] & \text { in } \Omega \\
w & =0 & & \text { on } \Gamma .
\end{array}\right.
$$

Since the right hand side of the equation belongs to $W^{-1, p}(\Omega)$ and the linear equation (2.13) defines an isomorphism between $W_{0}^{1, p}(\Omega)$ and $W^{-1, p}(\Omega), w$ is well defined. Re-writing the last equation in the form

$$
\left\{\begin{aligned}
-\operatorname{div}\left[a(x, y) \nabla(w+\phi)+\frac{\partial a}{\partial y}(x, y) w \nabla z\right]+\frac{\partial f}{\partial y}(x, y) w & =0 \text { in } \Omega \\
w+\phi & =0 \text { on } \Gamma
\end{aligned}\right.
$$

and comparing this with $(3.15)$, we observe that $z_{w}=w+\phi$, therefore $\frac{\partial \mathcal{F}}{\partial y}(u, y) w=\phi$.

Next we define the discrete version of $\mathcal{F}$

$$
\mathcal{F}_{h}: L^{p}(\Omega) \times W_{0}^{1, p}(\Omega) \longrightarrow W_{0}^{1, p}(\Omega), \quad \mathcal{F}_{h}(u, y)=z_{h}-y
$$

where $z_{h}$ is the solution of the variational problem

$$
\left\{\begin{array}{l}
\text { Find } z_{h} \in Y_{h} \text { such that } \forall \phi_{h} \in Y_{h} \\
\int_{\Omega_{h}}\left[a(x, y(x)) \nabla z_{h} \nabla \phi_{h}+f(x, y(x)) \phi_{h}\right] \mathrm{d} x=\int_{\Omega_{h}} u \phi_{h} \mathrm{~d} x .
\end{array}\right.
$$

The following proposition states some important but evident properties of $\mathcal{F}_{h}$. Its proof is completely analogous to the previous one.

Proposition 3.5. The following properties hold true for $\mathcal{F}_{h}$ :

(1) $\mathcal{F}_{h}(u, y)=0$ if and only if $y \in Y_{h}$ and $y$ is a solution of (3.2).

(2) $\mathcal{F}_{h}$ is of class $C^{1}$ and the partial derivative of $\mathcal{F}_{h}$ with respect to $y$ is given by

$$
\frac{\partial \mathcal{F}_{h}}{\partial y}(u, y) w=z_{h}(w)-w
$$

where $z_{h}(w) \in Y_{h}$ satisfies $\forall \phi_{h} \in Y_{h}$

$$
\left.\int_{\Omega_{h}}\left\{\left[a(x, y) \nabla z_{h}(w)+\frac{\partial a}{\partial y}(x, y) w \nabla z_{h}\right] \nabla \phi_{h}+\frac{\partial f}{\partial y}(x, y) w \phi_{h}\right]\right\} \mathrm{d} x=0 .
$$

The next result states that $\frac{\partial \mathcal{F}}{\partial y}(\bar{u}, \bar{y})$ can be approximated as closely as we wish by $\frac{\partial \mathcal{F}_{h}}{\partial y}(u, y)$, provided that $h$ is sufficiently small and $u$ and $y$ are taken close enough to $\bar{u}$ and $\bar{y}$. 
Proposition 3.6. For any $\varepsilon>0$, there exist $h_{\varepsilon}>0, \rho_{\varepsilon, \bar{u}}>0$ and $\rho_{\varepsilon, \bar{y}}>0$ such that

$$
\left\|\frac{\partial \mathcal{F}_{h}}{\partial y}(u, y)-\frac{\partial \mathcal{F}}{\partial y}(\bar{u}, \bar{y})\right\|_{\mathcal{L}\left(W_{0}^{1, p}(\Omega)\right)}<\varepsilon
$$

for all $0<h \leq h_{\varepsilon},\|u-\bar{u}\|_{L^{p}(\Omega)} \leq \rho_{\varepsilon, \bar{u}}$ and $\|y-\bar{y}\|_{W_{0}^{1, p}(\Omega)} \leq \rho_{\varepsilon, \bar{y}}$.

Proof. We have to show that for any $w \in W_{0}^{1, p}(\Omega)$

$$
\left\|\left[\frac{\partial \mathcal{F}_{h}}{\partial y}(u, y)-\frac{\partial \mathcal{F}}{\partial y}(\bar{u}, \bar{y})\right] w\right\|_{W_{0}^{1, p}(\Omega)}<\varepsilon\|w\|_{W_{0}^{1, p}(\Omega)} .
$$

Notice that it holds

$$
\left[\frac{\partial \mathcal{F}_{h}}{\partial y}(u, y)-\frac{\partial \mathcal{F}}{\partial y}(\bar{u}, \bar{y})\right] w=\left(z_{h}(w)-w\right)-\left(\bar{z}_{w}-w\right)=z_{h}(w)-\bar{z}_{w}
$$

Let us list for convenience the functions we will handle and the equations that they solve. Take $\bar{y}, z, \bar{z}_{w}, z_{w} \in$ $W_{0}^{1, p}(\Omega) \cap W^{2, p}(\Omega)$ and $z_{h}, z_{h}(w), z_{w, h} \in Y_{h}$ satisfying

$$
\begin{aligned}
& -\operatorname{div}[a(x, \bar{y}) \nabla \bar{y}]=\bar{u}-f(x, \bar{y}) \\
& -\operatorname{div}[a(x, y) \nabla z]=u-f(x, y) \\
& -\operatorname{div}\left[a(x, \bar{y}) \nabla \bar{z}_{w}+\frac{\partial a}{\partial y}(x, \bar{y}) w \nabla \bar{y}\right]+\frac{\partial f}{\partial y}(x, \bar{y}) w=0 \\
& -\operatorname{div}\left[a(x, y) \nabla z_{w}+\frac{\partial a}{\partial y}(x, y) w \nabla z\right]+\frac{\partial f}{\partial y}(x, y) w=0 \\
& \int_{\Omega_{h}}\left\{a(x, y) \nabla z_{h} \nabla \phi_{h}\right\} \mathrm{d} x=\int_{\Omega_{h}}[u-f(x, y)] \phi_{h} \mathrm{~d} x \quad \forall \phi_{h} \in Y_{h} \\
& \int_{\Omega_{h}}\left\{\left[a(x, y) \nabla z_{h}(w)+\frac{\partial a}{\partial y}(x, y) w \nabla z_{h}\right] \nabla \phi_{h}+\frac{\partial f}{\partial y}(x, y) w \phi_{h}\right\} \mathrm{d} x=0 \quad \forall \phi_{h} \in Y_{h} \\
& \int_{\Omega_{h}}\left\{\left[a(x, y) \nabla z_{w, h}+\frac{\partial a}{\partial y}(x, y) w \nabla z\right] \nabla \phi_{h}+\frac{\partial f}{\partial y}(x, y) w \phi_{h}\right\} \mathrm{d} x=0 \quad \forall \phi_{h} \in Y_{h} .
\end{aligned}
$$

Notice that $z_{h}$ is the FEM approximation of $z$. We have to estimate the right hand side of (3.21). To do this, we insert some intermediate functions and obtain

$$
\left\|z_{h}(w)-\bar{z}_{w}\right\|_{W_{0}^{1, p}(\Omega)} \leq\left\|z_{h}(w)-z_{w, h}\right\|_{W_{0}^{1, p}(\Omega)}+\left\|z_{w, h}-z_{w}\right\|_{W_{0}^{1, p}(\Omega)}+\left\|z_{w}-\bar{z}_{w}\right\|_{W_{0}^{1, p}(\Omega)} .
$$

Next, we estimate each of these three terms. First we assume that $y$ and $u$ are chosen such that

$$
\|u-\bar{u}\|_{L^{p}(\Omega)} \leq 1 \text { and }\|y-\bar{y}\|_{W_{0}^{1, p}(\Omega)} \leq 1 .
$$

A smaller radius will be introduced later. Let us estimate the first term of the right hand side of (3.29). By subtracting the equations (3.27) and (3.28), for $z_{h}(w)$ and $z_{w, h}$ shifting $\partial a / \partial y$ to the right-hand sides, and using the results by Brenner and Scott [2], see also Rannacher and Scott [12], we get

$$
\begin{aligned}
\left\|z_{h}(w)-z_{w, h}\right\|_{W_{0}^{1, p}(\Omega)} \leq C \| \operatorname{div}\left[\frac{\partial a}{\partial y}(x, y) w \nabla\left(z_{h}-z\right)\right] & \|_{W^{-1, p}(\Omega)} \\
\leq C\left\|z_{h}-z\right\|_{W_{0}^{1, p}(\Omega)} & \|w\|_{L^{\infty}(\Omega)} \leq C h\|z\|_{W^{2, p}(\Omega)}\|w\|_{W_{0}^{1, p}(\Omega)}
\end{aligned}
$$

where $p^{\prime}$ is the conjugate of $p$. Notice that $y$ is bounded, and this property transfers to $\partial a / \partial y$. 
The estimation of the second term of (3.29) follows again from the results in $[2,12]$. Indeed it is enough to notice that (3.28) is the discretization of equation (3.25), therefore

$$
\left\|z_{w, h}-z_{w}\right\|_{W_{0}^{1, p}(\Omega)} \leq C h\left\|z_{w}\right\|_{W^{2, p}(\Omega)} \leq C h\left(1+\|z\|_{W^{2, p}(\Omega)}\right)\|w\|_{W_{0}^{1, p}(\Omega)} \leq C h\|w\|_{W_{0}^{1, p}(\Omega)} .
$$

Finally we estimate the last term of (3.29). Subtracting equations (3.25) and (3.24) we get

$$
\begin{aligned}
& -\operatorname{div}\left\{a(x, \bar{y}) \nabla\left(z_{w}-\bar{z}_{w}\right)\right\}=-\operatorname{div}\left\{[a(x, \bar{y})-a(x, y)] \nabla z_{w}\right\} \\
& -\operatorname{div}\left\{\left[\frac{\partial a}{\partial y}(x, \bar{y}) \nabla \bar{y}-\frac{\partial a}{\partial y}(x, y) \nabla z\right] w\right\}+\left[\frac{\partial f}{\partial y}(x, \bar{y})-\frac{\partial f}{\partial y}(x, y)\right] w,
\end{aligned}
$$

hence

$$
\left\|z_{w}-\bar{z}_{w}\right\|_{W_{0}^{1, p}(\Omega)} \leq C\left(\|y-\bar{y}\|_{W_{0}^{1, p}(\Omega)}+\|z-\bar{y}\|_{W_{0}^{1, p}(\Omega)}\right)\|w\|_{L^{\infty}(\Omega)} .
$$

Now from (3.23) and (3.22) we get

$$
-\operatorname{div}[a(x, \bar{y}) \nabla(z-\bar{y})]=-\operatorname{div}[(a(x, \bar{y})-a(x, y)) \nabla z]+(u-\bar{u})-(f(x, y)-f(x, \bar{y})),
$$

hence

$$
\|z-\bar{y}\|_{W_{0}^{1, p}(\Omega)} \leq C\left(\|u-\bar{u}\|_{L^{p}(\Omega)}+\|y-\bar{y}\|_{W_{0}^{1, p}(\Omega)}\right) .
$$

This inequality, along with (3.32), leads to

$$
\left\|z_{w}-\bar{z}_{w}\right\|_{W_{0}^{1, p}(\Omega)} \leq C\left(\|u-\bar{u}\|_{L^{p}(\Omega)}+\|y-\bar{y}\|_{W_{0}^{1, p}(\Omega)}\right)\|w\|_{L^{\infty}(\Omega)} .
$$

Collecting (3.30), (3.31), and (3.33) we conclude the theorem.

Corollary 3.7. If $\varepsilon$ is chosen by

$$
0<\varepsilon<\frac{1}{2}\left\|\frac{\partial \mathcal{F}}{\partial y}(\bar{u}, \bar{y})^{-1}\right\|_{\mathcal{L}\left(W_{0}^{1, p}(\Omega)\right)}^{-1},
$$

then

$$
\frac{\partial \mathcal{F}_{h}}{\partial y}(u, y): W_{0}^{1, p}(\Omega) \longrightarrow W_{0}^{1, p}(\Omega)
$$

is an isomorphism for every $h \leq h_{\varepsilon},\|u-\bar{u}\|_{L^{p}(\Omega)} \leq \rho_{\varepsilon, \bar{u}}$ and $\|y-\bar{y}\|_{W_{0}^{1, p}(\Omega)} \leq \rho_{\varepsilon, \bar{y}}$ and

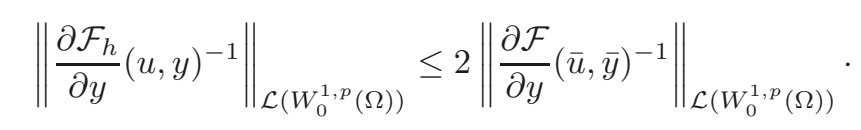

It is enough to combine Proposition 3.6 with well known results for operators in Banach spaces.

Finally, we arrive at the theorem on local existence and uniqueness of solutions of (3.2).

Theorem 3.8. There exist $h_{0}>0, \rho_{\bar{u}}>0$ and $\rho_{\bar{y}}>0$ such that the following holds: for any $h \leq h_{0}$ and $\|u-\bar{u}\|_{L^{p}(\Omega)} \leq \rho_{\bar{u}}$, the equation $\mathcal{F}_{h}(u, y)=0$ has in the ball $\bar{B}_{\rho_{\bar{y}}}(\bar{y})$ of $W_{0}^{1, p}(\Omega)$ a unique solution $y_{h}(u) \in Y_{h}$.

Proof. (i) Definition of a mapping $\Psi_{h}$. Select $\varepsilon$ by

$$
0<\varepsilon<\frac{1}{8}\left\|\frac{\partial \mathcal{F}}{\partial y}(\bar{u}, \bar{y})^{-1}\right\|_{\mathcal{L}\left(W_{0}^{1, p}(\Omega)\right)}^{-1}
$$


and fix $h_{\varepsilon}, \rho_{\varepsilon, \bar{u}}$ and $\rho_{\varepsilon, \bar{y}}$ as in Corollary 3.7. For $h \leq h_{\varepsilon}, 0<\rho_{1} \leq \rho_{\varepsilon, \bar{u}}, \rho_{\bar{y}}=\rho_{\varepsilon, \bar{y}}$, and $u \in \bar{B}_{\rho_{1}}(\bar{u}) \subset L^{p}(\Omega)$, we define a mapping $\psi_{u}: \bar{B}_{\rho_{\bar{y}}}(\bar{y}) \subset W_{0}^{1, p}(\Omega) \longrightarrow W_{0}^{1, p}(\Omega)$ by

$$
\psi_{u}(y)=y-\frac{\partial \mathcal{F}_{h}}{\partial y}(\bar{u}, \bar{y})^{-1} \mathcal{F}_{h}(u, y) .
$$

It is obvious that $\mathcal{F}_{h}(u, y)=0$ if and only if $y$ is a fixed point of $\psi_{u}$. We prove that $\psi_{u}$ is a contractive mapping from the ball $B_{\rho_{\bar{y}}}(\bar{y}) \subset W_{0}^{1, p}(\Omega)$ into itself, provided that $h_{0}$ and $\rho_{1}$ are sufficiently small. We define

$$
D(u, y):=\frac{\partial \mathcal{F}}{\partial y}(u, y), \quad \bar{D}:=D(\bar{u}, \bar{y}), \quad D_{h}(u, y):=\frac{\partial \mathcal{F}_{h}}{\partial y}(u, y), \quad \bar{D}_{h}:=D_{h}(\bar{u}, \bar{y})
$$

to shorten the next formulas.

(ii) An auxiliary inequality. First, we show the inequality

$$
\left\|\psi_{u}(y)-\bar{y}\right\|_{W_{0}^{1, p}(\Omega)} \leq \rho_{\bar{y}}
$$

for all $h, u, y$ with $h \leq h_{0},\|u-\bar{u}\|_{L^{p}(\Omega)} \leq \rho_{\bar{u}}$ and $\|y-\bar{y}\|_{W_{0}^{1, p}(\Omega)} \leq \rho_{\bar{y}}$. For such $u$ and $y$ we have

$$
\begin{aligned}
& \left\|\psi_{u}(y)-\bar{y}\right\|_{W_{0}^{1, p}(\Omega)}=\left\|\bar{D}_{h}^{-1}\left\{\bar{D}_{h}(y-\bar{y})+\left[\mathcal{F}_{h}(u, \bar{y})-\mathcal{F}_{h}(u, y)\right]-\mathcal{F}_{h}(u, \bar{y})\right\}\right\|_{W_{0}^{1, p}(\Omega)} \\
& \leq 2\left\|\bar{D}^{-1}\right\|_{\mathcal{L}\left(W_{0}^{1, p}(\Omega)\right)}\left\{\left\|\mathcal{F}_{h}(u, \bar{y})\right\|_{W_{0}^{1, p}(\Omega)}\right. \\
& \left.+\left\|\bar{D}_{h}(y-\bar{y})+\int_{0}^{1} D_{h}(u, \bar{y}+\theta(y-\bar{y}))(\bar{y}-y) \mathrm{d} \theta\right\|_{W_{0}^{1, p}(\Omega)}\right\} \\
& \leq 2\left\|\bar{D}^{-1}\right\|_{\mathcal{L}\left(W_{0}^{1, p}(\Omega)\right)}\left\{\left\|\mathcal{F}_{h}(u, \bar{y})\right\|_{W_{0}^{1, p}(\Omega)}\right. \\
& \left.+\int_{0}^{1}\left\|\bar{D}_{h}-D_{h}(u, \bar{y}+\theta(y-\bar{y}))\right\|_{\mathcal{L}\left(W_{0}^{1, p}(\Omega)\right)} \mathrm{d} \theta\|y-\bar{y}\|_{W_{0}^{1, p}(\Omega)}\right\} \\
& \leq 2\left\|\bar{D}^{-1}\right\|_{\mathcal{L}\left(W_{0}^{1, p}(\Omega)\right)}\left\{\left\|\mathcal{F}_{h}(u, \bar{y})\right\|_{W_{0}^{1, p}(\Omega)}+\left(\left\|\bar{D}_{h}-\bar{D}\right\|_{\mathcal{L}\left(W_{0}^{1, p}(\Omega)\right)}\right.\right. \\
& \left.\left.+\int_{0}^{1}\left\|\bar{D}-D_{h}(u, \bar{y}+\theta(\bar{y}-y))\right\|_{\mathcal{L}\left(W_{0}^{1, p}(\Omega)\right)} \mathrm{d} \theta\right)\|y-\bar{y}\|_{W_{0}^{1, p}(\Omega)}\right\} \\
& \leq 2\left\|\bar{D}^{-1}\right\|_{\mathcal{L}\left(W_{0}^{1, p}(\Omega)\right)}\left\{\left\|\mathcal{F}_{h}(u, \bar{y})\right\|_{W_{0}^{1, p}(\Omega)}+2 \varepsilon\|y-\bar{y}\|_{W_{0}^{1, p}(\Omega)}\right\} .
\end{aligned}
$$

The last inequality is a consequence of Proposition 3.6 and our choice for $h, \rho_{1}$ and $\rho_{\bar{y}}$. From the definition of $\mathcal{F}_{h}$ we know that $\mathcal{F}_{h}(u, \bar{y})=z_{h}-\bar{y}$, where $z_{h} \in Y_{h}$ satisfies

$$
\int_{\Omega_{h}}\left\{a(x, \bar{y}) \nabla z_{h} \cdot \nabla \phi_{h}\right\} \mathrm{d} x=\int_{\Omega_{h}}[u-f(x, \bar{y})] \phi_{h} \mathrm{~d} x \quad \forall \phi_{h} \in Y_{h} .
$$


Let us define $z \in W_{0}^{1, p}(\Omega) \cap W^{2, p}(\Omega)$ by

$$
-\operatorname{div}[a(x, \bar{y}) \nabla z]=u-f(x, \bar{y})
$$

Then $z_{h}$ is the finite element approximation of $z$. Making the difference between the equations satisfied by $\bar{y}$ and $\bar{z}$ (cf. the proof of Prop. 3.6), we get

$$
\left\|\mathcal{F}_{h}(u, \bar{y})\right\|_{W_{0}^{1, p}(\Omega)}=\left\|z_{h}-\bar{y}\right\|_{W_{0}^{1, p}(\Omega)} \leq\left\|z_{h}-z\right\|_{W_{0}^{1, p}(\Omega)}+\|z-\bar{y}\|_{W_{0}^{1, p}(\Omega)} \leq C\left(h+\|u-\bar{u}\|_{L^{p}(\Omega)}\right) .
$$

Notice that $\|z-\bar{y}\|_{W_{0}^{1, p}(\Omega)} \leq c\|u-\bar{u}\|_{L^{p}(\Omega)}$. Combining (3.37) and (3.38) we deduce

$$
\left\|\psi_{u}(y)-\bar{y}\right\|_{W_{0}^{1, p}(\Omega)} \leq 2\left\|\bar{D}^{-1}\right\|_{\mathcal{L}\left(W_{0}^{1, p}(\Omega)\right)}\left\{C\left(h+\|u-\bar{u}\|_{L^{p}(\Omega)}\right)+2 \varepsilon\|y-\bar{y}\|_{W_{0}^{1, p}(\Omega)}\right\} .
$$

By choosing $0<h_{0} \leq h_{\varepsilon}$ and $0<\rho_{\bar{u}} \leq \rho_{\varepsilon, \bar{u}}$ such that

$$
h_{0}+\rho_{\bar{u}} \leq \frac{\rho_{\bar{y}}}{4 C}\left\|\bar{D}^{-1}\right\|_{\mathcal{L}\left(W_{0}^{1, p}(\Omega)\right)}^{-1},
$$

we get from (3.35) and (3.39) the desired inequality (3.36).

(iii) Contractivity of $\psi_{u}: \bar{B}_{\rho_{\bar{y}}}(\bar{y}) \longrightarrow \bar{B}_{\rho_{\bar{y}}}(\bar{y})$. Take $y_{1}, y_{2} \in \bar{B}_{\rho_{2}}(\bar{y})$. Then

$$
\begin{array}{r}
\left\|\psi_{u}\left(y_{2}\right)-\psi_{u}\left(y_{1}\right)\right\|_{W_{0}^{1, p}(\Omega)}=\left\|\bar{D}_{h}^{-1}\left\{D_{h}(\bar{u}, \bar{y})\left(y_{2}-y_{1}\right)-\left[\mathcal{F}_{h}\left(u, y_{2}\right)-\mathcal{F}_{h}\left(u, y_{1}\right)\right]\right\}\right\|_{W_{0}^{1, p}(\Omega)} \\
\leq 2\left\|D_{h}(\bar{u}, \bar{y})^{-1}\right\|_{\mathcal{L}\left(W_{0}^{1, p}(\Omega)\right)}\left\|D_{h}(\bar{u}, \bar{y})\left(y_{2}-y_{1}\right)-\int_{0}^{1} D_{h}\left(u, y_{1}+\theta\left(y_{2}-y_{1}\right)\right)\left(y_{2}-y_{1}\right) \mathrm{d} \theta\right\|_{W_{0}^{1, p}(\Omega)} \\
\leq 2\left\|\bar{D}^{-1}\right\|_{\mathcal{L}\left(W_{0}^{1, p}(\Omega)\right)} \int_{0}^{1}\left\|D_{h}(\bar{u}, \bar{y})-D_{h}\left(u, y_{1}+\theta\left(y_{2}-y_{1}\right)\right)\right\|_{\mathcal{L}\left(W_{0}^{1, p}(\Omega)\right)} \mathrm{d} \theta\left\|y_{2}-y_{1}\right\|_{W_{0}^{1, p}(\Omega)} \\
\leq 4\left\|\bar{D}^{-1}\right\|_{\mathcal{L}_{\left(W_{0}^{1, p}(\Omega)\right)} \varepsilon\left\|y_{2}-y_{1}\right\|_{W_{0}^{1, p}(\Omega)}}
\end{array}
$$

The last inequality follows from Proposition 3.6 in the following way

$$
\begin{aligned}
\left\|D_{h}(\bar{u}, \bar{y})-D_{h}\left(u, y_{1}+\theta\left(y_{2}-y_{1}\right)\right)\right\|_{\mathcal{L}\left(W_{0}^{1, p}(\Omega)\right)} \leq & \left\|D_{h}(\bar{u}, \bar{y})-D(\bar{u}, \bar{y})\right\|_{\mathcal{L}\left(W_{0}^{1, p}(\Omega)\right)}+\| D(\bar{u}, \bar{y}) \\
& -D_{h}\left(u, y_{1}+\theta\left(y_{2}-y_{1}\right)\right) \|_{{\mathcal{L}\left(W_{0}^{1, p}(\Omega)\right)}<2 \varepsilon .}
\end{aligned}
$$

Finally (3.35) and (3.40) lead to

$$
\left\|\psi_{u}\left(y_{2}\right)-\psi_{u}\left(y_{1}\right)\right\|_{W_{0}^{1, p}(\Omega)} \leq \frac{1}{2}\left\|y_{2}-y_{1}\right\|_{W_{0}^{1, p}(\Omega)}
$$

therefore $\psi_{u}$ is contractive.

\subsection{Error estimates in $W^{1, p}(\Omega)$}

To derive $L^{p}$-error estimates, we follow the standard procedure and establish at first an estimate in the $W^{1, p}$-norm. From this estimate, we obtain the $L^{p}$ estimate in the next subsection.

Let $h_{0}>0, \rho_{\bar{u}}>0$ and $\rho_{\bar{y}}>0$ be given by Theorem 3.8 and let us fix $n<p \leq \bar{p}$. Given $h<h_{0}$ and $u \in \bar{B}_{\rho_{\bar{u}}}(\bar{u}) \subset L^{p}(\Omega), y_{h}(u)$ will denote the unique solution of $(3.2)$ in the ball $\bar{B}_{\rho_{\bar{y}}}(\bar{y}) \subset W_{0}^{1, p}(\Omega)$. The existence and uniqueness of $y_{h}(u)$ is a consequence of Proposition 3.5, statement (1), and Theorem 3.8. Our next objective is to prove the following error estimate: 
Theorem 3.9. For all $u \in B_{\rho_{\bar{u}}}(\bar{u}) \subset L^{p}(\Omega)$, there holds the estimate

$$
\left\|y_{u}-y_{h}(u)\right\|_{W^{1, p}(\Omega)} \leq C(\bar{u}) h
$$

with some constant $C(\bar{u})$ that may depend on $\bar{u}$ but not on $h$.

Let us start with the following lemma.

Lemma 3.10. For $u \in B_{\rho_{\bar{u}}}(\bar{u})$ and $h \leq h_{0}$ the following inequality holds:

$$
\left\|y_{u}-y_{h}(u)\right\|_{W^{1, p}(\Omega)} \leq \frac{8}{3}\left\|\frac{\partial \mathcal{F}}{\partial y}(\bar{u}, \bar{y})^{-1}\right\|_{\mathcal{L}\left(W_{0}^{1, p}(\Omega)\right)}\left\|\mathcal{F}_{h}\left(u, y_{u}\right)\right\|_{W_{0}^{1, p}(\Omega)} .
$$

Proof. We adopt the definition of $D$ and $D_{h}$ from the last proof. Taking into account that $\mathcal{F}_{h}\left(u, y_{h}(u)\right)=0$ and using (3.34), we get

$$
\begin{aligned}
\left\|y_{u}-y_{h}(u)\right\|_{W^{1, p}(\Omega)}= & \left\|D_{h}(\bar{u}, \bar{y})^{-1}\left\{D_{h}(\bar{u}, \bar{y})\left(y_{u}-y_{h}(u)\right)-\left[\mathcal{F}_{h}\left(u, y_{u}\right)-\mathcal{F}_{h}\left(u, y_{h}(u)\right)\right]+\mathcal{F}_{h}\left(u, y_{u}\right)\right\}\right\|_{W_{0}^{1, p}(\Omega)} \\
\leq & 2\left\|D(\bar{u}, \bar{y})^{-1}\right\|_{\mathcal{L}\left(W_{0}^{1, p}(\Omega)\right)}\left\{\left\|\mathcal{F}_{h}\left(u, y_{u}\right)\right\|_{W_{0}^{1, p}(\Omega)}\right. \\
& \left.+\int_{0}^{1}\left\|D_{h}(\bar{u}, \bar{y})-D_{h}\left(u, y_{h}(u)+\theta\left(y_{u}-y_{h}(u)\right)\right)\right\|_{\mathcal{L}_{\left(W_{0}^{1, p}(\Omega)\right)}} \mathrm{d} \theta\left\|y_{u}-y_{h}(u)\right\|_{W_{0}^{1, p}(\Omega)}\right\} .
\end{aligned}
$$

Using (3.19) and taking into account that $h_{0}, \rho_{\bar{u}}$ and $\rho_{\bar{y}}$ were chosen for $\varepsilon$ satisfying (3.35), we deduce from the previous inequality

$$
\left\|y_{u}-y_{h}(u)\right\|_{W^{1, p}(\Omega)} \leq 2\left\|D(\bar{u}, \bar{y})^{-1}\right\|_{\mathcal{L}\left(W_{0}^{1, p}(\Omega)\right)}\left\|\mathcal{F}_{h}\left(u, y_{u}\right)\right\|_{W_{0}^{1, p}(\Omega)}+\frac{1}{4}\left\|y_{u}-y_{h}(u)\right\|_{W_{0}^{1, p}(\Omega)},
$$

which implies (3.41).

Proof of Theorem 3.9. We prove estimate (3.41) by Lemma 3.10. Recalling the definition of $\mathcal{F}$ and $\mathcal{F}_{h}$, we find that $\mathcal{F}\left(u, y_{u}\right)=0$ and

$$
\mathcal{F}_{h}\left(u, y_{u}\right)=\mathcal{F}_{h}\left(u, y_{u}\right)-\mathcal{F}\left(u, y_{u}\right)=\left(z_{h}-y_{u}\right)-\left(z-y_{u}\right)=z_{h}-z
$$

where $z \in W_{0}^{1, p}(\Omega)$ and $z_{h} \in Y_{h}$ satisfy

$$
-\operatorname{div}\left[a\left(x, y_{u}\right) \nabla z\right]=u-f\left(x, y_{u}\right)
$$

and

$$
\int_{\Omega_{h}}\left\{a\left(x, y_{u}\right) \nabla z_{h} \cdot \nabla \phi_{h}\right\} \mathrm{d} x=\int_{\Omega_{h}}\left[u-f\left(x, y_{u}\right)\right] \phi_{h} \mathrm{~d} x \quad \forall \phi_{h} \in Y_{h} .
$$

From well known estimates for the finite element approximation of elliptic partial differential equations, see [2, $12]$, we get that

$$
\left\|z-z_{h}\right\|_{W_{0}^{1, p}(\Omega)} \leq C h\|z\|_{W^{2, p}(\Omega)} .
$$

By (3.43), this gives an estimate for $\left\|\mathcal{F}_{h}\left(u, y_{u}\right)\right\|$. Combining this with (3.42), we deduce (3.41). 


\subsection{Error estimates in $L^{p}(\Omega)$}

To start with the error estimates in $L^{p}$, we notice first that the linear operator

$$
\frac{\partial \mathcal{F}}{\partial y}(u, y): W_{0}^{1, p}(\Omega) \longrightarrow W_{0}^{1, p}(\Omega)
$$

can be extended to $L^{p}(\Omega)$ in the following way

$$
\frac{\widehat{\partial \mathcal{F}}}{\partial y}(u, y): L^{p}(\Omega) \longrightarrow L^{p}(\Omega), \quad \frac{\widehat{\partial \mathcal{F}}}{\partial y}(u, y) w=z_{w}-w
$$

where $z_{w}$ is the solution of (3.15). Since we take $w$ in $L^{p}(\Omega)$ instead of $W_{0}^{1, p}(\Omega)$ as we did in Proposition 3.4, we have that $z_{w} \in W_{0}^{1, p}(\Omega)$, but in general it is not an element of $W^{2, p}(\Omega)$. Let us verify that $\frac{\widehat{\partial \mathcal{F}}}{\partial y}(u, y)$ is an isomorphism for any $u \in L^{p}(\Omega)$ and $y \in W_{0}^{1, p}(\Omega)$. Repeating the proof of Proposition 3.4, statement (3), we confirm that $\frac{\widehat{\partial \mathcal{F}}}{\partial y}(u, y)$ is injective. For the surjectivity, we have to show that, for all $\phi \in L^{p}(\Omega)$, the equation

$$
z_{w}-w=\phi
$$

is solvable. To do this, we define $\hat{z} \in W_{0}^{1, p}(\Omega)$ by

$$
-\operatorname{div}\left\{a(x, y) \nabla \hat{z}+\frac{\partial a}{\partial y}(x, y) \hat{z} \nabla z\right\}+\frac{\partial f}{\partial y}(x, y) \hat{z}=-\operatorname{div}\left\{\frac{\partial a}{\partial y}(x, y) \phi \nabla z\right\}+\frac{\partial f}{\partial y}(x, y) \phi \text { in } \Omega
$$

where $z$ is the solution of (3.13). The right hand side of the equation is an element of $W^{-1, p}(\Omega)$. Because $(2.13)$ defines an isomorphism between $W_{0}^{1, p}(\Omega)$ and $W^{-1, p}(\Omega)$, we deduce that $\hat{z}$ is well defined. Now we write the previous equation in the form

$$
-\operatorname{div}\left\{a(x, y) \nabla \hat{z}+\frac{\partial a}{\partial y}(x, y)(\hat{z}-\phi) \nabla z\right\}+\frac{\partial f}{\partial y}(x, y)(\hat{z}-\phi)=0 \text { in } \Omega
$$

and set $w=\hat{z}-\phi$. Then we deduce from the previous equation and (3.15) that $\hat{z}=z_{w}$ and therefore $\frac{\widehat{\partial \mathcal{F}}}{\partial y}(u, y) w=\hat{z}-w=\phi$; hence the surjectivity is shown.

Analogously, we can extend $\frac{\partial \mathcal{F}_{h}}{\partial y}(u, y)$ to $L^{p}(\Omega)$ by

$$
\frac{\widehat{\partial \mathcal{F}_{h}}}{\partial y}(u, y): L^{p}(\Omega) \longrightarrow L^{p}(\Omega), \quad \frac{\widehat{\partial \mathcal{F}_{h}}}{\partial y}(u, y) w=z_{h}(w)-w
$$

where $z_{h}(w) \in Y_{h}$ is the solution of (3.18). As in Proposition 3.6, we find that for any $\varepsilon>0$ there exist $h_{\varepsilon}>0$, $\rho_{\varepsilon, \bar{u}}>0$, and $\rho_{\varepsilon, \bar{y}}>0$ such that

$$
\left\|\frac{\widehat{\partial \mathcal{F}_{h}}}{\partial y}(u, y)-\frac{\widehat{\partial \mathcal{F}}}{\partial y}(\bar{u}, \bar{y})\right\|_{\mathcal{L}\left(L^{p}(\Omega)\right)}<\varepsilon
$$

for $h \leq h_{\varepsilon}, u \in \bar{B}_{\rho_{\varepsilon, \bar{u}}}(\bar{u})$ and $y \in \bar{B}_{\rho_{\varepsilon, \bar{y}}}(\bar{y})$.

Notice that (3.44) is the $L^{p}$-counterpart of (3.19). Therefore, to show (3.44), we proceed as in the proof of Proposition 3.6 with the following changes: Inequality (3.29) is written in the form

$$
\left\|z_{h}(w)-\bar{z}_{w}\right\|_{L^{p}(\Omega)} \leq\left\|z_{h}(w)-z_{w, h}\right\|_{L^{p}(\Omega)}+\left\|z_{w, h}-z_{w}\right\|_{L^{p}(\Omega)}+\left\|z_{w}-\bar{z}_{w}\right\|_{L^{p}(\Omega)}
$$


By using that $p>n, W_{0}^{1, \frac{n p}{n+p}}(\Omega) \subset L^{p}(\Omega)$, and the error estimates of the finite element method [2,12], we change (3.31) by

$$
\begin{aligned}
& \left\|z_{h}(w)-z_{w, h}\right\|_{L^{p}(\Omega)} \leq C\left\|z_{h}(w)-z_{w, h}\right\|_{W_{0}^{1, \frac{n p}{n+p}}(\Omega)} \leq C\left\|\operatorname{div}\left[\frac{\partial a}{\partial y}(x, y) w \nabla\left(z_{h}-z\right)\right]\right\|_{W^{-1, \frac{n p}{n+p}(\Omega)}} \\
& \quad \leq C\left\|\frac{\partial a}{\partial y}(x, y) w \nabla\left(z_{h}-z\right)\right\|_{L^{\frac{n p}{n+p}(\Omega)}} \leq C\left\|z-z_{h}\right\|_{W_{0}^{1, p}(\Omega)}\|w\|_{L^{n}(\Omega)} \leq C h\|z\|_{W^{2, p}(\Omega)}\|w\|_{L^{p}(\Omega)} \leq C h\|w\|_{L^{p}(\Omega)} .
\end{aligned}
$$

Now (3.31) can be replaced by

$$
\left\|z_{w, h}-z_{w}\right\|_{L^{p}(\Omega)} \leq C h\left\|z_{w}\right\|_{W_{0}^{1, p}(\Omega)} \leq C h\|w\|_{L^{p}(\Omega)} .
$$

The inequalities (3.32) are transformed as follows:

$$
\begin{aligned}
\left\|z_{w}-\bar{z}_{w}\right\|_{L^{p}(\Omega)} \leq C\left\|z_{w}-\bar{z}_{w}\right\|_{W_{0}^{1, \frac{n p}{n+p}}(\Omega)} \leq & C\left\|\operatorname{div}\left\{[a(x, \bar{y})-a(x, y)] \nabla z_{w}\right\}\right\|_{W^{-1, \frac{n p}{n+p}}(\Omega)} \\
& +\left\|\operatorname{div}\left\{\left[\frac{\partial a}{\partial y}(x, \bar{y}) \nabla \bar{y}-\frac{\partial a}{\partial y}(x, y) \nabla z\right] w\right\}\right\|_{W^{-1, \frac{n p}{n+p}(\Omega)}} \\
& +\left\|\left[\frac{\partial f}{\partial y}(x, \bar{y})-\frac{\partial f}{\partial y}(x, y)\right] w\right\|_{W^{-1, \frac{n p}{n+p}}(\Omega)} \\
\leq & C\left\{\|y-\bar{y}\|_{L^{\infty}(\Omega)}\left\|\nabla z_{w}\right\|_{L^{\frac{n p}{n+p}}(\Omega)}+\left[\|y-\bar{y}\|_{W_{0}^{1, p}(\Omega)}\right.\right. \\
& \left.\left.+\|z-\bar{y}\|_{W_{0}^{1, p}(\Omega)}\right]\|w\|_{L^{n}(\Omega)}\right\} \\
\leq & C\left\{\|y-\bar{y}\|_{W_{0}^{1, p}(\Omega)}+\|z-\bar{y}\|_{W_{0}^{1, p}(\Omega)}\right\}\|w\|_{L^{p}(\Omega)} .
\end{aligned}
$$

We complete the proof of (3.44) in the way we finished the proof of Proposition 3.6 after (3.22).

Also Corollary 3.7 remains valid for $\frac{\widehat{\partial \mathcal{F}}_{h}}{\partial y}(u, y)$ if we replace $W_{0}^{1, p}(\Omega)$ by $L^{p}(\Omega)$. Therefore we find as in Lemma 3.10

$$
\left\|y_{u}-y_{h}(u)\right\|_{L^{p}(\Omega)} \leq \frac{8}{3}\left\|\frac{\widehat{\partial \mathcal{F}}}{\partial y}(\bar{u}, \bar{y})^{-1}\right\|_{\mathcal{L}\left(L^{p}(\Omega)\right)}\left\|\mathcal{F}_{h}\left(u, y_{u}\right)\right\|_{L^{p}(\Omega)} .
$$

Finally we can argue as in the proof of Theorem 3.9 to deduce

$$
\left\|y_{u}-y_{h}(u)\right\|_{L^{p}(\Omega)} \leq C\left\|z-z_{h}\right\|_{L^{p}(\Omega)} \leq C h^{2} .
$$

\section{Numerical ANALYSis OF THE ADJOINT STATE EQUATION}

In this section, our goal is to carry out the numerical analysis of the adjoint equation

$$
\left\{\begin{aligned}
-\operatorname{div}[a(x, y) \nabla \varphi]+\frac{\partial a}{\partial y}(x, y) \nabla y \cdot \nabla \varphi+\frac{\partial f}{\partial y}(x, y) \varphi & =v \text { in } \Omega \\
\varphi & =0 \text { on } \Gamma
\end{aligned}\right.
$$

for any $v \in L^{p}(\Omega)$, with $2 \leq p \leq \bar{p}$, where $y=y_{u} \in W^{2, \bar{p}}(\Omega)$ is the solution of (1.1) associated to $u \in L^{\bar{p}}(\Omega)$. This analysis is divided into two parts. First we consider a discretization of equation (4.1) by finite elements, but fixing $y$ in the discrete equation. Second, we replace $y$ by $y_{h}=y_{h}(u)$ in the discretized equation, to obtain the complete discretization of (4.1). 


\subsection{A partial discretization of equation (4.1)}

Throughout this section, we assume that $y \in W^{1, \infty}(\Omega)$. According to Remark 2.6, (4.1) defines an isomorphism between $W_{0}^{1, p}(\Omega)$ and $W^{-1, p}(\Omega)$. Moreover, if $v$ belongs to $L^{p}(\Omega)$ with $2 \leq p \leq \bar{p}$, then $\varphi$ belongs to $W^{2, p}(\Omega)$.

In the finite element space $Y_{h}$ defined upon $\mathcal{T}_{h}$, we approximate $\varphi$ by the solutions $\varphi_{h}$ of:

$$
\left\{\begin{array}{l}
\text { Find } \varphi_{h} \in Y_{h} \text { such that } \\
\int_{\Omega_{h}}\left\{a(x, y) \nabla \varphi_{h} \cdot \nabla \phi_{h}+\left[\frac{\partial a}{\partial y}(x, y) \nabla y \cdot \nabla \varphi_{h}+\frac{\partial f}{\partial y}(x, y) \varphi_{h}\right] \phi_{h}\right\} \mathrm{d} x=\int_{\Omega_{h}} v \phi_{h} \mathrm{~d} x \quad \forall \phi_{h} \in Y_{h} .
\end{array}\right.
$$

Since the operator above is not monotone, existence and/or uniqueness of a solution are not obvious. To deal with this lack of monotonicity we invoke an argument similar to the one used in Section 3.

We consider the linear mapping

$$
T: W_{0}^{1, p}(\Omega) \longrightarrow W_{0}^{1, p}(\Omega), \quad T w=\psi_{w}-w,
$$

where $\psi_{w} \in W_{0}^{1, p}(\Omega) \cap W^{2, p}(\Omega)$ satisfies the linear elliptic equation

$$
\left\{\begin{aligned}
-\operatorname{div}\left[a(x, y) \nabla \psi_{w}\right]+\frac{\partial a}{\partial y}(x, y) \nabla y \cdot \nabla w+\frac{\partial f}{\partial y}(x, y) w & =0 \text { in } \Omega \\
\psi_{w} & =0 \text { on } \Gamma .
\end{aligned}\right.
$$

Arguing as in Proposition 3.4 and using that (4.1) defines an isomorphism $v \leftrightarrow \varphi$ between $W_{0}^{1, p}(\Omega)$ and $W^{-1, p}(\Omega)$, see Remark 2.6, we deduce that $T$ is an isomorphism. Now we define the $Y_{h}$-version of $T$,

$$
T_{h}: W_{0}^{1, p}(\Omega) \longrightarrow W_{0}^{1, p}(\Omega), \quad T_{h} w=\psi_{h}(w)-w,
$$

where $\psi_{h}(w) \in Y_{h}$ satisfies $\forall \phi_{h} \in Y_{h}$

$$
\int_{\Omega_{h}}\left\{a(x, y) \nabla \psi_{h}(w) \cdot \nabla \phi_{h}+\left[\frac{\partial a}{\partial y}(x, y) \nabla y \cdot \nabla w+\frac{\partial f}{\partial y}(x, y) w\right] \phi_{h}\right\} \mathrm{d} x=0 .
$$

Invoking the $W^{1, p}(\Omega)$-error estimate of the finite element method for linear elliptic equations $[2,12]$, we get for any $w \in W_{0}^{1, p}(\Omega)$

$$
\left\|\left(T-T_{h}\right) w\right\|_{W_{0}^{1, p}(\Omega)}=\left\|\psi_{w}-\psi_{h}(w)\right\|_{W_{0}^{1, p}(\Omega)} \leq C h\left\|\psi_{w}\right\|_{W^{2, p}(\Omega)} \leq C h\|w\|_{W_{0}^{1, p}(\Omega)} .
$$

Notice that the term [...] in (4.4) is a fixed element of $L^{p}(\Omega)$, since $\partial a / \partial y$ and $\nabla y$ are bounded. The last inequality implies

$$
\left\|T-T_{h}\right\|_{\mathcal{L}\left(W_{0}^{1, p}(\Omega)\right)} \leq C h .
$$

The constant $C$ may depend on $y$, but because $y$ is bounded independently of $u \in U_{a d}$, we have a uniform bound. For this constant $C$, we select $\hat{h}_{0}>0$ such that

$$
\hat{h}_{0}<\left\{2 C\left\|T^{-1}\right\|_{\mathcal{L}\left(W_{0}^{1, p}(\Omega)\right.}\right\}^{-1} .
$$

Then $T_{h}$ is an isomorphism and

$$
\left\|T_{h}^{-1}\right\|_{\mathcal{L}\left(W_{0}^{1, p}(\Omega)\right)} \leq 2\left\|T^{-1}\right\|_{\mathcal{L}\left(W_{0}^{1, p}(\Omega)\right)} \quad \forall h<\hat{h}_{0} .
$$


Now we can prove existence and uniqueness of a solution of (4.2).

Theorem 4.1. For any $h \leq \hat{h}_{0}$ and every $v \in W^{-1, p}(\Omega), 2 \leq p \leq \bar{p}$, the variational problem (4.2) has a unique solution $\varphi_{h} \in Y_{h}$ and there exists a constant $C>0$ independent of $h$ such that

$$
\left\|\varphi_{h}\right\|_{W_{0}^{1, p}(\Omega)} \leq C\|v\|_{W^{-1, p}(\Omega)} .
$$

If $v$ is not an element of $L^{p}(\Omega)$, then the integral in the right hand side of $(4.2)$ must be replaced by the duality $\left\langle v, \phi_{h}\right\rangle$ between $W^{-1, p}(\Omega)$ and $W_{0}^{1, p^{\prime}}(\Omega)$.

Proof. Let us first prove the existence of at least one solution $\varphi_{h}$ of (4.2). We introduce $z_{h} \in Y_{h}$ such that

$$
\int_{\Omega_{h}} a(x, y) \nabla z_{h} \cdot \nabla \phi_{h} \mathrm{~d} x=\left\langle v, \phi_{h}\right\rangle \quad \forall \phi_{h} \in Y_{h}
$$

Since $T_{h}$ is an isomorphism, there exists a unique element $w \in W_{0}^{1, p}(\Omega)$ such that $T_{h} w=-z_{h}$, or equivalently $w=\psi_{h}(w)+z_{h}$. Now it is obvious that $w$ belongs to $Y_{h}$ and from (4.4) and (4.9) we deduce that $\varphi_{h}=w$ is a solution of (4.2).

To verify the inequality (4.8), we note that there exists a constant $C$ independent of $h$ such that

$$
\left\|z_{h}\right\|_{W_{0}^{1, p}(\Omega)} \leq C\|v\|_{W^{-1, p}(\Omega)}
$$

see [2]. On the other hand, as $\psi_{h}(w)$ is the finite element approximation of the solution $\psi_{w}$ to (4.3),

$$
\begin{gathered}
\left\|\psi_{h}(w)\right\|_{W_{0}^{1, p}(\Omega)} \leq C\left\|\psi_{w}\right\|_{W_{0}^{1, p}(\Omega)} \leq C\|w\|_{W_{0}^{1, p}(\Omega)}=C\left\|T_{h}^{-1} T_{h} w\right\|_{W_{0}^{1, p}(\Omega)} \\
\leq 2 C\left\|T^{-1}\right\|_{\mathcal{L}\left(W_{0}^{1, p}(\Omega)\right)}\left\|T_{h} w\right\|_{W_{0}^{1, p}(\Omega)}=2 C\left\|T^{-1}\right\|_{\mathcal{L}\left(W_{0}^{1, p}(\Omega)\right)}\left\|z_{h}\right\|_{W_{0}^{1, p}(\Omega)} .
\end{gathered}
$$

The first estimate in this chain is a consequence of the estimates of [2] or [12], while the second follows from (4.3). This inequality, along with the previous one and the definition of $\varphi_{h}$, proves (4.8).

Finally, we show the uniqueness. Assume that $\varphi_{h}$ satisfies (4.2) with right-hand side $v=0$ and set $w=\varphi_{h}$. Then we have that $\psi_{h}(w)=\varphi_{h}$ and then $T_{h} w=\varphi_{h}-\varphi_{h}=0$, therefore $w=0$ holds.

Next we estimate the error in $W_{0}^{1, p}(\Omega)$.

Theorem 4.2. For any $h \leq \hat{h}_{0}$ and $v \in L^{p}(\Omega), 2 \leq p \leq \bar{p}$, it holds

$$
\left\|\varphi-\varphi_{h}\right\|_{W_{0}^{1, p}(\Omega)} \leq C h\|v\|_{L^{p}(\Omega)}
$$

where $\varphi$ and $\varphi_{h}$ are the solutions of (4.1) and (4.2), respectively.

Proof. Using (4.5) and (4.7) we get

$$
\begin{aligned}
\left\|\varphi-\varphi_{h}\right\|_{W_{0}^{1, p}(\Omega)}= & \left\|T_{h}^{-1} T_{h}\left(\varphi-\varphi_{h}\right)\right\|_{W_{0}^{1, p}(\Omega)} \leq 2\left\|T^{-1}\right\|_{\mathcal{L}\left(W_{0}^{1, p}(\Omega)\right)}\left\|T_{h}\left(\varphi-\varphi_{h}\right)\right\|_{W_{0}^{1, p}(\Omega)} \\
& \leq 2\left\|T^{-1}\right\|_{\mathcal{L}\left(W_{0}^{1, p}(\Omega)\right)}\left\{\left\|\left(T_{h}-T\right) \varphi\right\|_{W_{0}^{1, p}(\Omega)}+\left\|T \varphi-T_{h} \varphi_{h}\right\|_{W_{0}^{1, p}(\Omega)}\right\} \\
& \leq 2\left\|T^{-1}\right\|_{\mathcal{L}\left(W_{0}^{1, p}(\Omega)\right)}\left\{C h\|\varphi\|_{W_{0}^{1, p}(\Omega)}+\left\|T \varphi-T_{h} \varphi_{h}\right\|_{W_{0}^{1, p}(\Omega)}\right\} .
\end{aligned}
$$

In view of the definition of $T$ and $T_{h}$ and equations (4.1) to (4.4), after setting $z=-T \varphi=\varphi-\psi_{\varphi}$ and $z_{h}=-T_{h} \varphi_{h}=\varphi_{h}-\psi_{h}\left(\varphi_{h}\right)$, we see that $z_{h}$ fulfills $(4.9)$ and $z \in W_{0}^{1, p}(\Omega) \cap W^{2, p}(\Omega)$ obeys

$$
-\operatorname{div}[a(x, y) \nabla z]=v \quad \text { in } \Omega .
$$


Therefore, it holds

$$
\left\|T \varphi-T_{h} \varphi_{h}\right\|_{W_{0}^{1, p}(\Omega)}=\left\|z-z_{h}\right\|_{W_{0}^{1, p}(\Omega)} \leq C h\|z\|_{W^{2, p}(\Omega)} \leq C h\|v\|_{L^{p}(\Omega)}
$$

This inequality, along with (4.11) and the estimate for $\varphi$ in terms of the norm of $v$ in $W^{-1, p}(\Omega)$ implies $(4.10)$.

We finish this section by estimating the error of $\varphi-\varphi_{h}$ in the $L^{p}(\Omega)$-norm. To achieve this goal we proceed along the lines of Section 3. We consider an extension of $T$ to $L^{p}(\Omega)$ denoted by $\hat{T}: L^{p}(\Omega) \longrightarrow L^{p}(\Omega)$,

$$
\hat{T} w=\psi_{w}-w
$$

where $\psi_{w}$ is the solution of (4.3). We should remark that $\psi_{w}$ is not in general an element of $W^{2, p}(\Omega)$. Arguing as in the case of $\frac{\widehat{\partial \mathcal{F}}}{\partial y}(u, y)$, we deduce that $\hat{T}$ is still an isomorphism. Analogously we extend $T_{h}$ to $L^{p}(\Omega)$ and we have, see $[2,12]$,

$$
\left\|\left(\hat{T}-\hat{T}_{h}\right) w\right\|_{L^{p}(\Omega)}=\left\|\psi_{w}-\psi_{h}(w)\right\|_{L^{p}(\Omega)} \leq C h\left\|\psi_{w}\right\|_{W_{0}^{1, p}(\Omega)} \leq C h\|w\|_{L^{p}(\Omega)}
$$

which implies

$$
\left\|\hat{T}-\hat{T}_{h}\right\|_{\mathcal{L}\left(L^{p}(\Omega)\right)} \leq C h .
$$

For $w \in W_{0}^{1, p}(\Omega)$, we have $\psi_{w} \in W^{2, p}(\Omega)$ and hence we can improve the above estimate by

$$
\left\|\left(\hat{T}-\hat{T}_{h}\right) w\right\|_{L^{p}(\Omega)}=\left\|\psi_{w}-\psi_{h}(w)\right\|_{L^{p}(\Omega)} \leq C h^{2}\left\|\psi_{w}\right\|_{W^{2, p}(\Omega)} \leq C h^{2}\|w\|_{W_{0}^{1, p}(\Omega)} .
$$

By (4.12), we also deduce an inequality analogous to (4.7),

$$
\left\|\hat{T}_{h}^{-1}\right\|_{\mathcal{L}\left(L^{p}(\Omega)\right)} \leq 2\left\|\hat{T}^{-1}\right\|_{\mathcal{L}\left(L^{p}(\Omega)\right)}
$$

We can modify the inequalities of (4.11) by the previous inequalities and get

$$
\begin{aligned}
\left\|\varphi-\varphi_{h}\right\|_{L^{p}(\Omega)}= & \left\|\hat{T}_{h}^{-1} \hat{T}_{h}\left(\varphi-\varphi_{h}\right)\right\|_{L^{p}(\Omega)} \leq 2\left\|\hat{T}^{-1}\right\|_{\mathcal{L}\left(L^{p}(\Omega)\right)}\left\|\hat{T}_{h}\left(\varphi-\varphi_{h}\right)\right\|_{L^{p}(\Omega)} \\
& \leq 2\left\|\hat{T}^{-1}\right\|_{\mathcal{L}\left(L^{p}(\Omega)\right)}\left\{\left\|\left(\hat{T}_{h}-\hat{T}\right) \varphi\right\|_{L^{p}(\Omega)}+\left\|T \varphi-T_{h} \varphi_{h}\right\|_{L^{p}(\Omega)}\right\} \\
& \leq 2\left\|\hat{T}^{-1}\right\|_{\mathcal{L}\left(W_{0}^{1, p}(\Omega)\right)}\left\{C h^{2}\|\varphi\|_{W_{0}^{1, p}(\Omega)}+\left\|\hat{T} \varphi-\hat{T}_{h} \varphi_{h}\right\|_{L^{p}(\Omega)}\right\} .
\end{aligned}
$$

Finally, taking $z=-T \varphi$ and $z_{h}=-T_{h} \varphi_{h}$ as in the proof of Theorem 4.2 , we find

$$
\left\|\hat{T} \varphi-\hat{T}_{h} \varphi_{h}\right\|_{L^{p}(\Omega)}=\left\|\hat{z}-z_{h}\right\|_{L^{p}(\Omega)} \leq C h^{2}\|z\|_{W^{2, p}(\Omega)} \leq C h^{2}\|v\|_{L^{p}(\Omega)} .
$$

The last two inequalities yields the desired result, which is stated in the next theorem.

Theorem 4.3. For any $h \leq \hat{h}_{0}$ and $v \in L^{p}(\Omega)$ we have

$$
\left\|\varphi-\varphi_{h}\right\|_{L^{p}(\Omega)} \leq C h^{2}\|v\|_{L^{p}(\Omega)}
$$

Remark 4.4. Many of the constants $C$ that appeared in this subsection depend on the norm $\|y\|_{W^{1, \infty}(\Omega)}$, but they can be chosen independently of the concrete form of $y$. 


\subsection{The full discretization of equation (4.1)}

In this section, $\bar{u} \in L^{\bar{p}}(\Omega)$ is a fixed locally optimal control with associated state $\bar{y} \in W^{2, \bar{p}}(\Omega) \cap W_{0}^{1, \bar{p}}(\Omega)$, a reference control for which we discretize the adjoint equation. According to Theorem 3.8, there exist $h_{0}>0$, $\rho_{\bar{u}}>0$ and $\rho_{\bar{y}}>0$ such that, for every $h \leq h_{0}$ and all $u \in \bar{B}_{\rho_{\bar{u}}}(\bar{u}) \subset L^{\bar{p}}(\Omega)$, equation (3.2) has in $Y_{h} \cap \bar{B}_{\rho_{\bar{y}}}(\bar{y})$ a unique solution $y_{h}(u)$. Here, $\bar{B}_{\rho_{\bar{y}}}(\bar{y})$ is the closed ball in $W_{0}^{1, \bar{p}}(\Omega)$. Let us denote by $y$ the state $y_{u}$ associated to a fixed element $u \in \bar{B}_{\rho_{\bar{u}}}(\bar{u})$ and by $y_{h}$ the discrete state $y_{h}(u)$. Then, according to Theorem 3.9 we also have that $y \in \bar{B}_{\rho_{\bar{y}}}(\bar{y}) \cap W^{2, \bar{p}}(\Omega)$.

We also assume that $h_{0} \leq \hat{h}_{0}$, where $\hat{h}_{0}$ is defined in (4.6).

Here, we consider the fully discretized equation associated with equation (4.1),

$$
\left\{\begin{array}{l}
\text { Find } \varphi_{h} \in Y_{h} \text { such that } \\
\int_{\Omega_{h}}\left\{a\left(x, y_{h}\right) \nabla \varphi_{h} \cdot \nabla \phi_{h}+\left[\frac{\partial a}{\partial y}\left(x, y_{h}\right) \nabla y_{h} \cdot \nabla \varphi_{h}+\frac{\partial f}{\partial y}\left(x, y_{h}\right) \varphi_{h}\right] \phi_{h}\right\} \mathrm{d} x=\int_{\Omega_{h}} v \phi_{h} \mathrm{~d} x \quad \forall \phi_{h} \in Y_{h} .
\end{array}\right.
$$

The goal of this section is to estimate $\varphi-\varphi_{h}$, where $\varphi$ is the solution of (4.1). Throughout this section, we assume that $v \in L^{p}(\Omega)$ for $n<p \leq \bar{p}$. To derive these estimates we introduce $\varphi^{h} \in W^{2, p}(\Omega) \subset C^{1}(\bar{\Omega})$, the solution of

$$
\left\{\begin{aligned}
-\operatorname{div}\left[a\left(x, y_{h}\right) \nabla \varphi^{h}\right]+\frac{\partial a}{\partial y}\left(x, y_{h}\right) \nabla y_{h} \cdot \nabla \varphi^{h}+\frac{\partial f}{\partial y}\left(x, y_{h}\right) \varphi^{h} & =v \text { in } \Omega \\
\varphi^{h} & =0 \text { on } \Gamma .
\end{aligned}\right.
$$

This is the adjoint equation (4.1), but now with $y_{h}$ substituted for $y$.

We prove the following result:

Theorem 4.5. Assume $n \leq p \leq \bar{p}$ and $h \leq h_{0}$. Then, for all $v \in L^{p}(\Omega)$, the solutions $\varphi$ and $\varphi_{h}$ to (4.1) and (4.17), respectively, obey the estimate

$$
\left\|\varphi-\varphi_{h}\right\|_{L^{p}\left(\Omega_{h}\right)}+h\left\|\varphi-\varphi_{h}\right\|_{W^{1, p}\left(\Omega_{h}\right)} \leq C h^{2}\|v\|_{L^{p}(\Omega)}
$$

Proof. (i) Estimation of $\varphi_{h}-\varphi^{h}$. First, we note that $\left\|y_{h}\right\|_{W^{1, \infty}(\Omega)}$ is bounded by a constant independently of $h$. Indeed, using the interpolation operator $\Pi_{h}: C(\bar{\Omega}) \rightarrow Y_{h}$, an inverse inequality, estimates (3.12) and well known properties of $\Pi_{h}$ we get

$$
\begin{aligned}
\left\|y_{h}\right\|_{W^{1, \infty}(\Omega)} & =\left\|y_{h}\right\|_{W^{1, \infty}\left(\Omega_{h}\right)} \leq\left\|y_{h}-\Pi_{h} y\right\|_{W^{1, \infty}\left(\Omega_{h}\right)}+\left\|\Pi_{h} y-y\right\|_{W^{1, \infty}\left(\Omega_{h}\right)}+\|y\|_{W^{1, \infty}\left(\Omega_{h}\right)} \\
& \leq C\left\{h^{-1-\frac{n}{\bar{p}}}\left\|y_{h}-\Pi_{h} y\right\|_{L^{\bar{p}}\left(\Omega_{h}\right)}+\left\|\Pi_{h} y-y\right\|_{W^{1, \infty}\left(\Omega_{h}\right)}\right\}+\|y\|_{W^{1, \infty}(\Omega)} \\
& \leq C\left\{h^{-1-\frac{n}{\bar{p}}}\left[\left\|y_{h}-y\right\|_{L^{\bar{p}}\left(\Omega_{h}\right)}+\left\|y-\Pi_{h} y\right\|_{L^{\bar{p}}\left(\Omega_{h}\right)}\right]+\left\|\Pi_{h} y-y\right\|_{W^{1, \infty}\left(\Omega_{h}\right)}\right\}+\|y\|_{W^{1, \infty}(\Omega)} \\
& \leq C\left(h^{1-\frac{n}{\bar{p}}}+1\right)\|y\|_{W^{2, \bar{p}}(\Omega)} .
\end{aligned}
$$

Since $\bar{p}>n$, we deduce the desired boundedness. Therefore, we can use Theorems 4.2 and 4.3 to deduce

$$
\left\|\varphi^{h}-\varphi_{h}\right\|_{L^{p}\left(\Omega_{h}\right)}+h\left\|\varphi^{h}-\varphi_{h}\right\|_{W^{1, p}\left(\Omega_{h}\right)} \leq C h^{2}\|v\|_{L^{p}(\Omega)}
$$

for any $h \leq h_{0}$. 
In view of this inequality, to estimate $\varphi-\varphi_{h}$, it is enough to consider $\varphi-\varphi^{h}$. Subtracting the equations (4.1) and (4.18) we obtain

$$
\begin{aligned}
& -\operatorname{div}\left[a(x, y)\left(\nabla \varphi-\nabla \varphi^{h}\right)\right]+\frac{\partial a}{\partial y}(x, y) \nabla y \cdot\left(\nabla \varphi-\nabla \varphi^{h}\right)+\frac{\partial f}{\partial y}(x, y)\left(\varphi-\varphi^{h}\right) \\
& =-\operatorname{div}\left[\left(a\left(x, y_{h}\right)-a(x, y)\right) \nabla \varphi^{h}\right]+\left[\frac{\partial a}{\partial y}\left(x, y_{h}\right) \nabla y_{h}-\frac{\partial a}{\partial y}(x, y) \nabla y\right] \nabla \varphi^{h}+\left[\frac{\partial f}{\partial y}\left(x, y_{h}\right)-\frac{\partial f}{\partial y}(x, y)\right] \varphi^{h} .
\end{aligned}
$$

(ii) Estimation of the right-hand side of (4.21) in $W^{-1, p}(\Omega)$. We mention first that the boundedness of $\left\{y_{h}\right\}_{h<h_{0}}$ in $W^{1, \infty}(\Omega)$ implies the boundedness of $\left\{\varphi^{h}\right\}_{h<h_{0}}$ in $W^{2, p}(\Omega) \subset C^{1}(\bar{\Omega})$. The bound for $\|\varphi\|_{W^{2, p}(\Omega)}$ depends on $\|v\|_{L^{p}(\Omega)}$. Hence, from (2.5) we deduce

$$
\left\|\left[\frac{\partial f}{\partial y}\left(x, y_{h}\right)-\frac{\partial f}{\partial y}(x, y)\right] \varphi^{h}\right\|_{L^{p}(\Omega)} \leq C\left\|y-y_{h}\right\|_{L^{p}(\Omega)} \leq C h^{2}\|v\|_{L^{p}(\Omega)} .
$$

The last inequality is obtained as follows: Consider the splitting

$$
\left\|y-y_{h}\right\|_{L^{p}(\Omega)} \leq\left\|y-y_{h}\right\|_{L^{p}\left(\Omega_{h}\right)}+\|y\|_{L^{p}\left(\Omega \backslash \Omega_{h}\right)} .
$$

Inequality (3.12) provides the estimate for the first item. For the second, we take into account that for any $x \in \Omega \backslash \Omega_{h}$ we can get a point $x_{\Gamma} \in \Gamma$ such that $\left|x-x_{\Gamma}\right| \leq C_{\Gamma} h^{2}$. Then we have for any $w \in W_{0}^{1, \infty}(\Omega)$

$$
|w(x)|=\left|w(x)-w\left(x_{\Gamma}\right)\right| \leq\|w\|_{W^{1, \infty}(\Omega)}\left|x-x_{\Gamma}\right| \leq C_{\Gamma}\|w\|_{W^{1, \infty}(\Omega)} h^{2},
$$

therefore

this yields (4.22).

$$
\|y\|_{L^{p}\left(\Omega \backslash \Omega_{h}\right)} \leq C\|y\|_{W^{1, \infty}(\Omega)} h^{2}\left|\Omega \backslash \Omega_{h}\right|^{1 / p} \leq C h^{2} ;
$$

For the first item in the right-hand side of (4.21), we get from (2.1)

$$
\begin{aligned}
\left\|\operatorname{div}\left[\left(a\left(x, y_{h}\right)-a(x, y)\right) \nabla \varphi^{h}\right]\right\|_{W^{-1, p}(\Omega)} & \leq\left\|\left(a\left(x, y_{h}\right)-a(x, y)\right) \nabla \varphi^{h}\right\|_{L^{p}(\Omega)} \\
& \leq C\left\|y-y_{h}\right\|_{L^{p}(\Omega)}\|v\|_{L^{p}(\Omega)} \leq C h^{2}\|v\|_{L^{p}(\Omega)} .
\end{aligned}
$$

Finally, we estimate the last item of the right-hand side,

$$
\begin{aligned}
\left\|\left[\frac{\partial a}{\partial y}\left(x, y_{h}\right) \nabla y_{h}-\frac{\partial a}{\partial y}(x, y) \nabla y\right] \nabla \varphi^{h}\right\|_{W^{-1, p}(\Omega)} \leq & \left\|\left[\frac{\partial a}{\partial y}\left(x, y_{h}\right)-\frac{\partial a}{\partial y}(x, y)\right] \nabla y_{h} \nabla \varphi^{h}\right\|_{W^{-1, p}(\Omega)} \\
& +\left\|\frac{\partial a}{\partial y}(x, y)\left(\nabla y_{h}-\nabla y\right) \nabla \varphi^{h}\right\|_{W^{-1, p}(\Omega)} .
\end{aligned}
$$

The first term is handled by (2.1), the boundedness of $y_{h}$ and $\varphi^{h}$ in $W^{1, \infty}(\Omega)$ and the estimate (3.12),

$$
\begin{aligned}
\left\|\left[\frac{\partial a}{\partial y}\left(x, y_{h}\right)-\frac{\partial a}{\partial y}(x, y)\right] \nabla y_{h} \nabla \varphi^{h}\right\|_{W^{-1, p}(\Omega)} & \leq\left\|\left[\frac{\partial a}{\partial y}\left(x, y_{h}\right)-\frac{\partial a}{\partial y}(x, y)\right] \nabla y_{h} \nabla \varphi^{h}\right\|_{L^{p}(\Omega)} \\
& \leq C\left\|y-y_{h}\right\|_{L^{p}(\Omega)}\|v\|_{L^{p}(\Omega)} \leq C h^{2}\|v\|_{L^{p}(\Omega)} .
\end{aligned}
$$

For the second term, we proceed as follows: First we note that, thanks to Assumption (H2), the mapping

$$
x \in \Omega \mapsto \frac{\partial a}{\partial y}(x, y(x)) \in \mathbb{R}
$$


belongs to $W^{1, \infty}(\Omega)$. On the other hand, we have $\nabla \varphi^{h} \in W^{1, p}(\Omega)^{n}$. Therefore, the function

$$
g^{h}(x):=\frac{\partial a}{\partial y}(x, y(x)) \nabla \varphi^{h}(x)
$$

is contained in $W^{1, p}(\Omega)^{n}$. Now, take an arbitrary $w \in W_{0}^{1, p^{\prime}}(\Omega)$ arbitrary, where $p^{\prime}=p /(p-1)$. Since $W_{0}^{1, p^{\prime}}(\Omega) \subset L^{\frac{n p^{\prime}}{n-p^{\prime}}}(\Omega)$ and the conjugate number of $\frac{n p^{\prime}}{n-p^{\prime}}$ is $\frac{n p}{n+p}$, we have

$$
\begin{gathered}
\left|\left\langle\frac{\partial a}{\partial y}(x, y)\left(\nabla y_{h}-\nabla y\right) \nabla \varphi^{h}, w\right\rangle_{W^{-1, p}(\Omega), W_{0}^{1, p^{\prime}}(\Omega)}\right|=\left|\int_{\Omega} g^{h}\left(\nabla y_{h}-\nabla y\right) w \mathrm{~d} x\right| \\
=\left|-\int_{\Omega}\left(\operatorname{div} g^{h}\right)\left(y_{h}-y\right) w \mathrm{~d} x-\int_{\Omega} g^{h}\left(y_{h}-y\right) \nabla w \mathrm{~d} x\right| \\
\leq\left\|\left(\operatorname{div} g^{h}\right)\left(y_{h}-y\right)\right\|_{L^{\frac{n p}{n+p}}(\Omega)}\|w\|_{L^{\frac{n p^{\prime}}{n-p^{\prime}}(\Omega)}}+\left\|g^{h}\right\|_{L^{\infty}(\Omega)}\left\|y_{h}-y\right\|_{L^{p}(\Omega)}\|\nabla w\|_{L^{p^{\prime}}(\Omega)} \\
\leq C\left\|\operatorname{div} g^{h}\right\|_{L^{\frac{2 n p}{n+p}}(\Omega)}\left\|y_{h}-y\right\|_{L^{\frac{2 n p}{n+p}}(\Omega)}\|w\|_{W^{1, p^{\prime}}(\Omega)}+\left\|g^{h}\right\|_{L^{\infty}(\Omega)}\|y h-y\|_{L^{p}(\Omega)}\|w\|_{W^{1, p^{\prime}}(\Omega)} \\
\leq C\left\|y_{h}-y\right\|_{L^{p}(\Omega)}\|v\|_{L^{p}(\Omega)}\|w\|_{W^{1, p^{\prime}}(\Omega)} \leq C h^{2}\|v\|_{L^{p}(\Omega)}\|w\|_{W^{1, p^{\prime}}(\Omega)} .
\end{gathered}
$$

We have used above that $p \geq n$ and hence $\frac{2 n p}{n+p} \leq p$.

Finally, since the adjoint state equation defines an isomorphism between $W_{0}^{1, p}(\Omega)$ and $W^{-1, p}(\Omega)$ (see Rem. 2.6), we deduce from (4.21)-(4.25) that

$$
\left\|\varphi-\varphi^{h}\right\|_{W^{1, p}(\Omega)} \leq C h^{2}\|v\|_{L^{p}(\Omega)} .
$$

The estimates (4.20) and (4.26) imply the statement of the theorem.

\section{Discretization of the CONTRol Problem}

Finally, we come to our main goal, the convergence of discretized optimal controls as $h \rightarrow 0$. We show that any strict local solution $\bar{u}$ with associated state $\bar{y}$ can be approximated by a strongly converging sequence of local solutions $\bar{u}_{h}$ of the discrete problems $\left(\mathrm{P}_{h}\right)$.

To this aim, we fix a strict local reference solution $\bar{u} \in \mathcal{U}_{a d}$ in the sense of $L^{p}(\Omega), 1 \leq p \leq+\infty$. Strict local solution means that there exists $\varepsilon>0$ such that

$$
J(\bar{u})<J(u) \quad \forall u \in \mathcal{U}_{a d} \cap \bar{B}_{\varepsilon}(\bar{u}) \text {, with } u \neq \bar{u},
$$

where $\bar{B}_{\varepsilon}(\bar{u})$ is a closed ball in $L^{p}(\Omega)$.

Denote by $\bar{y}$ the associated state. To define the discrete problem, we need some further notation. We take $h_{0}>0, \rho_{\bar{u}}>0$ and $\rho_{\bar{y}}>0$ as in Theorem 3.2. Then we introduce a mapping $G_{h}: \bar{B}_{\rho_{\bar{u}}}(\bar{u}) \subset L^{\bar{p}}(\Omega) \longrightarrow$ $Y_{h} \cap \bar{B}_{\rho_{\bar{y}}}(\bar{y})$ by $G_{h}(u)=y_{h}(u)$ for every $h \leq h_{0}$. By applying the implicit function theorem to the mapping $\mathcal{F}_{h}$ we deduce with the help of Corollary 3.7 that $G_{h}$ is of class $C^{1}$ in $B_{\rho_{\bar{u}}}(\bar{u})$. Moreover, $z_{h}(v):=G_{h}^{\prime}(u) v$ is the solution of the following variational problem:

$$
\left\{\begin{array}{l}
\text { Find } z_{h}(v) \in Y_{h} \text { such that } \\
\int_{\Omega_{h}}\left[a\left(x, y_{h}(u)\right) \nabla z_{h}(v)+\frac{\partial a}{\partial y}\left(x, y_{h}(u)\right) z_{h}(v) \nabla y_{h}(u)\right] \nabla \phi_{h} \mathrm{~d} x \\
+\int_{\Omega_{h}} \frac{\partial f}{\partial y}\left(x, y_{h}(u)\right) z_{h}(v) \phi_{h} \mathrm{~d} x=\int_{\Omega_{h}} v \phi_{h} \mathrm{~d} x \quad \forall \phi_{h} \in Y_{h} .
\end{array}\right.
$$


We will assume that $\varepsilon$ is chosen so small such that (5.1) holds and

$$
0<\varepsilon<\left\{\begin{array}{cl}
\frac{\rho_{\bar{u}}}{|\Omega|^{1 / \bar{p}}} & \text { if } p=\infty \\
\frac{\rho_{\bar{u}}^{\bar{p}}}{(\beta-\alpha)^{\bar{p}-1}|\Omega|^{(p-1) / p}} & \text { if } p<\infty .
\end{array}\right.
$$

Then, for all $u \in \bar{B}_{\varepsilon}(\bar{u})$ and $p=\infty$, it holds

$$
\|u-\bar{u}\|_{L^{\bar{p}}(\Omega)} \leq \varepsilon|\Omega|^{1 / \bar{p}}<\rho_{\bar{u}} \quad \Rightarrow \quad u \in B_{\rho_{\bar{u}}}(\bar{u}) \subset L^{\bar{p}}(\Omega) .
$$

Moreover, we have for all $p<\infty$

$$
\begin{aligned}
\|u-\bar{u}\|_{L^{\bar{p}}(\Omega)} & \leq(\beta-\alpha)^{(\bar{p}-1) / \bar{p}}\|u-\bar{u}\|_{L^{1}(\Omega)}^{1 / \bar{p}} \leq(\beta-\alpha)^{(\bar{p}-1) / \bar{p}}|\Omega|^{(p-1) / p \bar{p}}\|u-\bar{u}\|_{L^{p}(\Omega)}^{1 / \bar{p}} \\
& \leq(\beta-\alpha)^{(\bar{p}-1) / \bar{p}}|\Omega|^{(p-1) / p \bar{p}} \varepsilon^{1 / \bar{p}}<\rho_{\bar{u}} .
\end{aligned}
$$

In either case, $u$ belongs to $B_{\rho_{\bar{u}}}(\bar{u}) \subset L^{\bar{p}}(\Omega)$.

For every $h>0$, let $\mathcal{U}_{h}$ be a subspace of $L^{\infty}\left(\Omega_{h}\right)$ and define

$$
\mathcal{U}_{a d, h}=\mathcal{U}_{h} \cap \mathcal{U}_{a d}=\left\{u_{h} \in \mathcal{U}_{h}: \alpha \leq u_{h}(x) \leq \beta \text { for a.e. } x \in \Omega_{h}\right\} .
$$

We assume the following approximation property:

(H5) For every $u \in U_{a d} \cap C^{0,1}(\bar{\Omega})$, there exists a sequence $\left\{u_{h}\right\}_{h>0}$ with $u_{h} \in \mathcal{U}_{a d, h}$ such that

$$
\lim _{h \rightarrow 0}\left\|u-u_{h}\right\|_{L^{\infty}\left(\Omega_{h}\right)}=0
$$

For every $h \leq h_{0}$, the discrete control problem is defined as follows:

$$
\left(\mathrm{P}_{h}^{\varepsilon}\right) \quad\left\{\begin{array}{l}
\min J_{h}\left(u_{h}\right):=\int_{\Omega_{h}} L\left(x, y_{h}\left(u_{h}\right)(x), u_{h}(x)\right) \mathrm{d} x \\
u_{h} \in \mathcal{U}_{a d, h} \cap \bar{B}_{\varepsilon}(\bar{u}),
\end{array}\right.
$$

where $y_{h}\left(u_{h}\right)=G_{h}\left(u_{h}\right) \in Y_{h} \cap \bar{B}_{\rho_{\bar{y}}}(\bar{y})$.

Strictly speaking, the functions $u_{h} \in \mathcal{U}_{h}$ are not defined in the whole domain $\Omega$, therefore the assumption $u_{h} \in \bar{B}_{\varepsilon}(\bar{u})$ is not entirely precise. However it is clear that the definitions of $y_{h}(u)$ and $J_{h}\left(u_{h}\right)$ only requires the values $u_{h}(x)$ for $x \in \Omega_{h}$. Therefore any extension of $u_{h}$ to $\Omega$ that remains in the ball $\bar{B}_{\varepsilon}(\bar{u})$ avoids this formal difficulty. In practice, we only compute the values of $u_{h}$ in $\Omega_{h}$ and we are interested in the behavior of these values when $h \rightarrow 0$. The simplest way of extending every element $u_{h} \in \mathcal{U}_{h}$ to $\Omega$ is the setting $u_{h}(x)=\bar{u}(x)$ for every $x \in \Omega \backslash \Omega_{h}$. This will be assumed in the sequel.

Common choices of $\mathcal{U}_{h}$ are piecewise constant controls or piecewise linear and continuous controls associated with the triangulation $\mathcal{T}_{h}$. In both of these cases, the assumption (H5) is satisfied for $p<\infty$ and in the first case it even holds for $p=\infty$. Moreover, also the choice of undiscretized controls $\mathcal{U}_{h}=L^{\infty}\left(\Omega_{h}\right)$ is interesting and useful in practical computations (cf. the concept of variational discretization by Hinze [8]), and (H5) is fulfilled for every $1 \leq p \leq \infty$.

Since $J_{h}$ is a continuous functional and the set of admissible controls $\mathcal{U}_{a d, h} \cap \bar{B}_{\varepsilon}(\bar{u})$ is compact in $\mathcal{U}_{h}$ and non-empty (at least for $h$ small enough), then $\left(\mathrm{P}_{h}^{\varepsilon}\right)$ has at least one optimal solution. To check that $\mathcal{U}_{a d, h} \cap \bar{B}_{\varepsilon}(\bar{u})$ is non-empty it is enough to notice that $\bar{u} \in C^{0,1}(\Omega)$ by Theorem 2.7 and to invoke hypothesis (H5). Then we get a sequence $\left\{u_{h}\right\}_{h>0}$ such that $u_{h} \in \mathcal{U}_{a d, h}$ and $\left\|\bar{u}-u_{h}\right\|_{L^{\infty}(\Omega)} \rightarrow 0$. Thus $u_{h} \in \mathcal{U}_{a d, h} \cap \bar{B}_{\varepsilon}(\bar{u})$ for every $h$ 
small enough. Let us formulate the optimality conditions satisfied by these local minima. Taking into account Theorem 4.1, we obtain the following results, which are the discrete counterparts of Theorems 2.4 and 2.5:

Theorem 5.1. For every $h \leq h_{0}$, the functional $J_{h}: B_{\rho_{\bar{u}}}(\bar{u}) \cap L^{\infty}(\Omega) \rightarrow \mathbb{R}$ is of class $C^{1}$, and its derivative is given by

$$
J_{h}^{\prime}(u) v=\int_{\Omega_{h}}\left(\frac{\partial L}{\partial u}\left(x, y_{h}(u), u\right)+\varphi_{h}(u)\right) v \mathrm{~d} x,
$$

where $\varphi_{h}(u) \in Y_{h}$ is the unique solution of the adjoint state equation

$$
\begin{aligned}
\int_{\Omega_{h}}\left[a\left(x, y_{h}(u)\right) \nabla \varphi_{h}(u) \cdot \nabla \phi_{h}+\frac{\partial a}{\partial y}\left(x, y_{h}(u)\right) \nabla y_{h}(u) \cdot \nabla \varphi_{h}(u) \phi_{h}\right] & \mathrm{d} x+\int_{\Omega_{h}} \frac{\partial f}{\partial y}\left(x, y_{h}(u)\right) \varphi_{h}(u) \phi_{h} \mathrm{~d} x \\
& =\int_{\Omega_{h}} \frac{\partial L}{\partial y}\left(x, y_{h}(u), u\right) \phi_{h} \mathrm{~d} x \quad \forall \phi_{h} \in Y_{h} .
\end{aligned}
$$

From this expression for the derivative, we deduce the first-order necessary optimality conditions for the discretized problem in a standard way.

Theorem 5.2. Let $\bar{u}_{h}$ be a local solution of $\left(\mathrm{P}_{h}^{\varepsilon}\right)$. Then there exist two functions $\bar{y}_{h}$ and $\bar{\varphi}_{h}$ in $Y_{h}$ such that

$$
\begin{gathered}
\int_{\Omega_{h}}\left[a\left(x, \bar{y}_{h}(x)\right) \nabla \bar{y}_{h} \cdot \nabla \phi_{h}+f\left(x, \bar{y}_{h}(x)\right) \phi_{h}\right] \mathrm{d} x=\int_{\Omega_{h}} \bar{u}_{h} \phi_{h} \mathrm{~d} x \quad \forall \phi_{h} \in Y_{h} \\
\int_{\Omega_{h}}\left[a\left(x, \bar{y}_{h}\right) \nabla \bar{\varphi}_{h} \cdot \nabla \phi_{h}+\frac{\partial a}{\partial y}\left(x, \bar{y}_{h}\right) \nabla \bar{y}_{h} \cdot \nabla \bar{\varphi}_{h} \phi_{h}\right] \mathrm{d} x+\int_{\Omega_{h}} \frac{\partial f}{\partial y}\left(x, \bar{y}_{h}\right) \bar{\varphi}_{h} \phi_{h} \mathrm{~d} x \\
=\int_{\Omega_{h}} \frac{\partial L}{\partial y}\left(x, \bar{y}_{h}, \bar{u}\right) \phi_{h} \mathrm{~d} x \quad \forall \phi_{h} \in Y_{h} \\
\int_{\Omega_{h}}\left(\frac{\partial L}{\partial u}\left(x, \bar{y}_{h}, \bar{u}_{h}\right)+\bar{\varphi}_{h}\right)\left(u_{h}-\bar{u}_{h}\right) \mathrm{d} x \geq 0 \quad \forall u_{h} \in \mathcal{U}_{a d, h} \cap \bar{B}_{\varepsilon}(\bar{u}) .
\end{gathered}
$$

From (5.9), we can derive explicit expressions for $\bar{u}_{h}$, if $\mathcal{U}_{h}$ is the space of piecewise constant functions or if $\mathcal{U}_{h}=L^{\infty}\left(\Omega_{h}\right)$. In the first case, arguing as in [1], we deduce from (5.9) that

$$
\bar{u}_{\left.h\right|_{T}}=\operatorname{Proj}_{\left[\alpha_{h, T}, \beta_{h, T}\right]}\left(\bar{s}_{\left.h\right|_{T}}\right) \quad \forall T \in \mathcal{T}_{h},
$$

where $s_{\left.h\right|_{T}}$ is the unique real number satisfying the equation

$$
\int_{T}\left(\frac{\partial L}{\partial u}\left(x, \bar{y}_{h}(x), \bar{s}_{\left.h\right|_{T}}\right)+\bar{\varphi}_{h}(x)\right) \mathrm{d} x=0
$$

and

$$
\alpha_{h, T}=\max \left\{\alpha, \max _{x \in T} \bar{u}(x)-\varepsilon\right\} \quad \text { and } \quad \beta_{h, T}=\min \left\{\beta, \min _{x \in T} \bar{u}(x)+\varepsilon\right\} .
$$

In the case $\mathcal{U}_{h}=L^{\infty}\left(\Omega_{h}\right)$, proceeding as in Theorem 2.7, we deduce from (5.9) that

$$
\bar{u}_{h}(x)=\operatorname{Proj}_{\left[\alpha^{\varepsilon}(x), \beta^{\varepsilon}(x)\right]}\left(\bar{s}_{h}(x)\right),
$$

where $\bar{s}_{h}(x)$ is the unique solution $t$ of the equation

$$
\frac{\partial L}{\partial u}\left(x, \bar{y}_{h}(x), t\right)+\bar{\varphi}_{h}(x)=0
$$


and

$$
\alpha^{\varepsilon}(x)=\max \{\alpha, \bar{u}(x)-\varepsilon\}, \quad \beta^{\varepsilon}(x)=\min \{\beta, \bar{u}(x)+\varepsilon\} .
$$

For piecewise linear controls, we do not have an analogous representation formula.

Now, we obtain a convergence result for the solutions of problems $\left(\mathrm{P}_{h}^{\varepsilon}\right)$. To this aim let us recall that all feasible controls $u$ of $\left(\mathrm{P}_{h}^{\varepsilon}\right)$ belong to $\bar{B}_{\varepsilon}(\bar{u}) \subset \bar{B}_{\rho_{\bar{u}}}(\bar{u})$ and, by definition, it holds $y_{h}(u) \in \bar{B}_{\rho_{\bar{y}}}(\bar{y})$; see Theorem 3.2, the definition of $G_{h}$ at the beginning of Section 5 and the definition of $\left(\mathrm{P}_{h}^{\varepsilon}\right)$.

Theorem 5.3. Let $\bar{u}_{h}, h \leq h_{0}$, be a family of solutions of $\left(\mathrm{P}_{h}^{\varepsilon}\right)$ and let $\bar{y}_{h}=y_{h}\left(\bar{u}_{h}\right)$. Then under the approximation assumption ( $\mathrm{H} 5)$ there holds the convergence property

$$
\lim _{h \rightarrow 0}\left\{\left\|\bar{u}-\bar{u}_{h}\right\|_{L^{2}\left(\Omega_{h}\right)}+\left\|\bar{y}-\bar{y}_{h}\right\|_{H^{1}\left(\Omega_{h}\right)}+\left\|\bar{y}-\bar{y}_{h}\right\|_{L^{\infty}\left(\Omega_{h}\right)}\right\}=0 .
$$

Moreover, if $\mathcal{U}_{h}=L^{\infty}\left(\Omega_{h}\right)$ or $\mathcal{U}_{h}$ is defined by piecewise constant controls, then also

$$
\lim _{h \rightarrow 0}\left\|\bar{u}-\bar{u}_{h}\right\|_{L^{\infty}\left(\Omega_{h}\right)}=0 .
$$

To prove this theorem, we need some preparatory lemmas.

Lemma 5.4. For every pair $u, v \in \mathcal{U}_{a d}$ with $v \in \bar{B}_{\rho_{\bar{u}}}(\bar{u})$, the following estimates hold:

$$
\begin{aligned}
& \left\|y_{u}-y_{h}(v)\right\|_{L^{2}\left(\Omega_{h}\right)}+\left\|\varphi_{u}-\varphi_{h}(v)\right\|_{L^{2}\left(\Omega_{h}\right)} \leq C\left(h^{2}+\|u-v\|_{L^{2}(\Omega)}\right) \\
& \left\|y_{u}-y_{h}(v)\right\|_{H^{1}\left(\Omega_{h}\right)}+\left\|\varphi_{u}-\varphi_{h}(v)\right\|_{H^{1}\left(\Omega_{h}\right)} \leq C\left(h+\|u-v\|_{L^{2}(\Omega)}\right) \\
& \left\|y_{u}-y_{h}(v)\right\|_{L^{\infty}\left(\Omega_{h}\right)}+\left\|\varphi_{u}-\varphi_{h}(v)\right\|_{L^{\infty}\left(\Omega_{h}\right)} \leq C\left(h+\|u-v\|_{L^{2}(\Omega)}\right) .
\end{aligned}
$$

Proof. First, we estimate $y_{u}-y_{h}(v)$. To show (5.15), we consider (3.3) and argue as follows:

$$
\left\|y_{u}-y_{h}(v)\right\|_{L^{2}\left(\Omega_{h}\right)} \leq\left\|y_{v}-y_{h}(v)\right\|_{L^{2}\left(\Omega_{h}\right)}+\left\|y_{u}-y_{v}\right\|_{L^{2}\left(\Omega_{h}\right)} \leq C\left(h^{2}+\|u-v\|_{L^{2}(\Omega)}\right) .
$$

The last term is obtained by the mean value theorem (see Rem. 2.3),

$$
\left\|y_{u}-y_{v}\right\|_{H^{2}(\Omega)} \leq \sup _{t \in[0,1]}\left\|G^{\prime}(v+t(u-v))\right\|_{\mathcal{L}\left(L^{2}(\Omega), H^{2}(\Omega)\right)}\|u-v\|_{L^{2}(\Omega)} .
$$

Analogously, we prove (5.16). To show (5.17), we apply inequality (3.12) in the following way:

$$
\begin{aligned}
\left\|y_{u}-y_{h}(v)\right\|_{L^{\infty}\left(\Omega_{h}\right)} & \leq\left\|y_{v}-y_{h}(v)\right\|_{L^{\infty}\left(\Omega_{h}\right)}+\left\|y_{u}-y_{v}\right\|_{L^{\infty}\left(\Omega_{h}\right)} \\
& \leq C\left(\left\|y_{v}-y_{h}(v)\right\|_{W^{1, \bar{p}}\left(\Omega_{h}\right)}+\left\|y_{u}-y_{v}\right\|_{H^{2}(\Omega)}\right) \leq C\left(h+\|u-v\|_{L^{2}(\Omega)}\right) .
\end{aligned}
$$

Finally, using (4.19), the estimates for the adjoint states follow by the same steps as above. The only difference is the estimation of $\varphi_{u}-\varphi_{v}$. For this purpose, we subtract the equations satisfied by both functions and get

$$
\begin{aligned}
-\operatorname{div} & {\left[a\left(x, y_{u}\right)\left(\nabla \varphi_{u}-\nabla \varphi_{v}\right)\right]+\frac{\partial a}{\partial y}\left(x, y_{u}\right) \nabla y_{u} \cdot\left(\nabla \varphi_{u}-\nabla \varphi_{v}\right)+\frac{\partial f}{\partial y}\left(x, y_{u}\right)\left(\varphi_{u}-\varphi_{v}\right) } \\
& =-\operatorname{div}\left[\left(a\left(x, y_{v}\right)-a\left(x, y_{u}\right)\right) \nabla \varphi_{v}\right]+\left[\frac{\partial a}{\partial y}\left(x, y_{v}\right) \nabla y_{v}-\frac{\partial a}{\partial y}\left(x, y_{u}\right) \nabla y_{u}\right] \nabla \varphi_{v}+\left[\frac{\partial f}{\partial y}\left(x, y_{v}\right)-\frac{\partial f}{\partial y}\left(x, y_{u}\right)\right] \varphi_{v}
\end{aligned}
$$

Now, we estimate $\left\|\varphi_{u}-\varphi_{v}\right\|_{H^{2}(\Omega)}$ by the $L^{2}(\Omega)$-norm of the right hand side. We use the assumptions (H2) and (H3) along with the above estimates for $y_{u}-y_{v}$. Moreover, we invoke the boundedness of $\varphi_{v}$ that is independent of $v$, since $v \in \mathcal{U}_{a d}$ and $\mathcal{U}_{a d}$ is a bounded subset of $L^{\infty}(\Omega)$, but it may depend on $\bar{u}$ and $\rho_{\bar{u}}$. Then it is a simple exercise to obtain that

$$
\left\|\varphi_{u}-\varphi_{v}\right\|_{H^{2}(\Omega)} \leq C\|u-v\|_{L^{2}(\Omega)} .
$$


Lemma 5.5. Let $u \in \mathcal{U}_{a d}$ be given and let a sequence $\left\{u_{h}\right\}_{h>0}$ with $u_{h} \in \mathcal{U}_{a d, h} \cap \bar{B}_{\rho_{\bar{u}}}(\bar{u})$ converge weakly to $u$,

$$
\lim _{h \rightarrow 0} \int_{\Omega_{h}} \phi\left(u-u_{h}\right) \mathrm{d} x=0 \quad \forall \phi \in L^{\infty}(\Omega) .
$$

Then it holds that

$$
\begin{aligned}
& \lim _{h \rightarrow 0}\left\{\left\|y_{u}-y_{h}\left(u_{h}\right)\right\|_{L^{\infty}\left(\Omega_{h}\right)}+\left\|y_{u}-y_{h}\left(u_{h}\right)\right\|_{H^{1}\left(\Omega_{h}\right)}\right\}=0, \\
& J(u) \leq \liminf _{h \rightarrow 0} J_{h}\left(u_{h}\right) .
\end{aligned}
$$

If, in addition,

$$
\lim _{h \rightarrow 0} \int_{\Omega_{h}}\left|u(x)-u_{h}(x)\right| \mathrm{d} x=0,
$$

then $\lim _{h \rightarrow 0} J_{h}\left(u_{h}\right)=J(u)$.

Proof. Recall that all elements $u_{h} \in \mathcal{U}_{h}$ were extended to $\Omega$ by $u_{h}(x)=\bar{u}(x)$ in $\Omega \backslash \Omega_{h}$. Therefore, $\left\{u_{h}\right\}_{h>0} \subset$ $\bar{B}_{\rho_{\bar{u}}}(\bar{u})$ is bounded in $L^{\bar{p}}(\Omega)$ and there exists a subsequence converging weakly in $L^{\bar{p}}(\Omega)$ to some element $\tilde{u} \in \bar{B}_{\rho_{\bar{u}}}(\bar{u})$. Relation (5.18) implies $\tilde{u}=u$, hence we have $u_{h} \rightarrow u$ in $L^{\bar{p}}(\Omega)$. Let us write $y_{h}=y_{h}\left(u_{h}\right)$ and $y=y_{u}$. From the definition of $y_{h}\left(u_{h}\right)=G_{h}\left(u_{h}\right)$ we find that $\left\{y_{h}\right\}_{h \leq h_{0}} \subset \bar{B}_{\rho_{\bar{y}}}(\bar{y}) \subset W_{0}^{1, \bar{p}}(\Omega)$, therefore there exists a subsequence converging weakly to some element $\tilde{y} \in \bar{B}_{\rho_{\bar{y}}}(\bar{y})$. The compactness of the inclusion $W_{0}^{1, \bar{p}}(\Omega) \subset L^{\infty}(\Omega)$ implies that this convergence is strong in $L^{\infty}(\Omega)$.

Now it is easy to pass to the limit in the equations satisfied by $y_{h}$ and to conclude that $\tilde{y}=y_{u}=y$. Moreover, we deduce by standard arguments that the convergence is strong in $H^{1}(\Omega)$. Since all the subsequences have the same limit, (5.19) holds. Inequality (5.20) follows as usual by the convexity of $L$ with respect to third variable.

The last assertion of the lemma is an immediate consequence of (5.19).

Proof of Theorem 5.3. We mention again that $\bar{u}_{h}(x)=\bar{u}(x)$ in $\Omega \backslash \Omega_{h}$. The uniform boundedness of $\left\{\bar{u}_{h}\right\}_{h>0}$ in $L^{\infty}(\Omega)$ yields the weak convergence $\bar{u}_{h} \rightarrow \tilde{u}$ in $L^{\bar{p}}(\Omega)$ to some $\tilde{u} \in \mathcal{U}_{a d} \cap \bar{B}_{\varepsilon}(\bar{u})$ for some subsequence denoted in the same way. Let us prove that $\tilde{u}=\bar{u}$. Taking into account that $\bar{u} \in C^{0,1}(\bar{\Omega})$ and assumption (H5), we deduce the existence of a sequence $\left\{u_{h}\right\}_{h>0}$ with $u_{h} \in \mathcal{U}_{a d, h}$ such that

$$
\lim _{h \rightarrow 0}\left\|\bar{u}-u_{h}\right\|_{L^{\infty}\left(\Omega_{h}\right)}=0 .
$$

The controls $u_{h}$ are admissible for $\left(\mathrm{P}_{h}^{\varepsilon}\right)$ for all sufficiently small $h$. By Lemma 5.5, we get

$$
J(\tilde{u}) \leq \liminf _{h \rightarrow 0} J_{h}\left(\bar{u}_{h}\right) \leq \limsup _{h \rightarrow 0} J_{h}\left(\bar{u}_{h}\right) \leq \limsup _{h \rightarrow 0} J_{h}\left(u_{h}\right)=J(\bar{u}) .
$$

These inequalities, along with (5.1), imply that $\tilde{u}=\bar{u}$ and

$$
\lim _{h \rightarrow 0} J_{h}\left(\bar{u}_{h}\right)=J(\bar{u})
$$

Once again Lemma 5.5 yields the convergence of the states $\left\{\bar{y}_{h}\right\}_{h>0}$ as claimed in (5.13). The convergence of the controls is proved as in [1], p. 223. From the identity (5.21) and assumption (2.6), the convergence in $L^{\infty}\left(\Omega_{h}\right)$ is got as in [1], pp. 223-224 when $\mathcal{U}_{h}$ is defined by piecewise constant functions.

In the case $\mathcal{U}_{h}=L^{\infty}\left(\Omega_{h}\right)$, the uniform convergence follows from the representations of $\bar{u}$ and $\bar{u}_{h}$ given by (2.22) and (5.12), along with the definitions of $\bar{s}$ and $\bar{s}_{h}$ as solutions of (2.21) and (5.11) and estimates provided in Lemma 5.4. We also use the fact that

$$
\alpha^{\varepsilon}(x) \leq \bar{u}(x)=\operatorname{Proj}_{[\alpha, \beta]}(\bar{s}(x)) \leq \beta^{\varepsilon}(x) \quad \Rightarrow \quad \bar{u}(x)=\operatorname{Proj}_{\left[\alpha^{\varepsilon}(x), \beta^{\varepsilon}(x)\right]}(\bar{s}(x)) .
$$


Now we proceed in the following way, using again the assumption (2.6) and the mean value theorem applied to the function $(\partial L / \partial u)\left(x, y_{h}(x), \cdot\right)$ : For any $x \in \Omega_{h}$,

$$
\begin{aligned}
\mid \bar{u}(x) & -\bar{u}_{h}(x)|=| \operatorname{Proj}_{\left[\alpha^{\varepsilon}(x), \beta^{\varepsilon}(x)\right]}(\bar{s}(x))-\operatorname{Proj}_{\left[\alpha^{\varepsilon}(x), \beta^{\varepsilon}(x)\right]}\left(\bar{s}_{h}(x)\right)|\leq| \bar{s}(x)-\bar{s}_{h}(x) \mid \\
& \leq \frac{1}{\Lambda}\left|\frac{\partial L}{\partial u}\left(x, \bar{y}_{h}(x), \bar{s}(x)\right)-\frac{\partial L}{\partial u}\left(x, \bar{y}_{h}(x), \bar{s}_{h}(x)\right)\right| \\
& \leq \frac{1}{\Lambda}\left\{\left|\frac{\partial L}{\partial u}\left(x, \bar{y}_{h}(x), \bar{s}(x)\right)-\frac{\partial L}{\partial u}(x, \bar{y}(x), \bar{s}(x))\right|+\left|\frac{\partial L}{\partial u}(x, \bar{y}(x), \bar{s}(x))-\frac{\partial L}{\partial u}\left(x, \bar{y}_{h}(x), \bar{s}_{h}(x)\right)\right|\right\} \\
& \leq \frac{1}{\Lambda}\left\{\left|\frac{\partial L}{\partial u}\left(x, \bar{y}_{h}(x), \bar{s}(x)\right)-\frac{\partial L}{\partial u}(x, \bar{y}(x), \bar{s}(x))\right|+\left\|\bar{\varphi}-\bar{\varphi}_{h}\right\|_{L^{\infty}\left(\Omega_{h}\right)}\right\} \rightarrow 0 .
\end{aligned}
$$

Since the controls $\left\{\bar{u}_{h}\right\}_{h>0}$ are uniformly bounded in $L^{\infty}(\Omega)$, the convergence $\bar{u}_{h} \rightarrow \bar{u}$ in $L^{2}(\Omega)$ implies the convergence in $L^{p}(\Omega)$ for all $p<\infty$. Hence $\bar{u}_{h}$ belongs to $B_{\varepsilon}(\bar{u})$ for all sufficiently small $h$. Therefore, the control $\bar{u}_{h}$ is a local minimum of problem

$$
\left(\mathrm{P}_{h}\right) \quad\left\{\begin{array}{l}
\min J_{h}\left(u_{h}\right):=\int_{\Omega_{h}} L\left(x, y_{h}(u)(x), u_{h}(x)\right) \mathrm{d} x \\
u_{h} \in \mathcal{U}_{a d, h}
\end{array}\right.
$$

for every sufficiently small $h$.

For $p=\infty$ the statement remains true if $\mathcal{U}_{h}=L^{\infty}\left(\Omega_{h}\right)$ or if $\mathcal{U}_{h}$ is space of the piecewise constant functions associated with the triangulation $\mathcal{T}_{h}$, because the convergence $\bar{u}_{h} \rightarrow \bar{u}$ is uniform in these two cases; see Theorem 5.3. In the case of piecewise linear and continuous controls, we can approximate strict local minima of (P) in the sense of the $L^{p}(\Omega)$-topology only if $p<\infty$. We do not know the answer for $p=\infty$. The difficulty is that a simple representation formula for $\bar{u}_{h}$ does not hold as in the other two cases.

Remark 5.6. The variational inequality (5.9) can be simplified. If $\bar{u}_{h}$ is a local minimum of $\left(\mathrm{P}_{h}\right)$, then (5.9) can be formulated as

$$
\int_{\Omega_{h}}\left(\frac{\partial L}{\partial u}\left(x, \bar{y}_{h}, \bar{u}_{h}\right)+\bar{\varphi}_{h}\right)\left(u_{h}-\bar{u}_{h}\right) \mathrm{d} x \geq 0 \quad \forall u_{h} \in \mathcal{U}_{a d, h}
$$

and the values $\alpha_{T, h}, \beta_{T, h}$ and $\alpha^{\varepsilon}, \beta^{\varepsilon}$ used in (5.10) and (5.11) can be replaced by $\alpha$ and $\beta$ respectively.

Based on our error estimates for the finite element approximation of the state and adjoint state equations, we have been able to prove the strong convergence of the discretized control problems. Another natural question is to estimate the distance of discretized controls to associated locally optimal controls of (P). This needs second order optimality conditions. Related error estimates will be derived in a forthcoming paper. Roughly speaking, we prove the following estimates:

If $\mathcal{U}_{h}$ consists of piecewise constant functions associated with the triangulation $\mathcal{T}_{h}$, then

$$
\left\|\bar{u}-\bar{u}_{h}\right\|_{L^{2}\left(\Omega_{h}\right)} \leq C h .
$$

If $\mathcal{U}_{h}$ is a set of piecewise linear continuous functions, then

$$
\left\|\bar{u}-\bar{u}_{h}\right\|_{L^{2}\left(\Omega_{h}\right)} \leq C h^{3 / 2} .
$$

Finally, for the case $\mathcal{U}_{h}=L^{\infty}\left(\Omega_{h}\right)$ it holds

$$
\left\|\bar{u}-\bar{u}_{h}\right\|_{L^{2}\left(\Omega_{h}\right)} \leq C h^{2} .
$$




\section{REFERENCES}

[1] N. Arada, E. Casas and F. Tröltzsch, Error estimates for the numerical approximation of a semilinear elliptic control problem. Comput. Optim. Appl. 23 (2002) 201-229.

[2] S.C. Brenner and L.R. Scott, The Mathematical Theory of Finite Element Methods. Springer-Verlag, New York-BerlinHeidelberg (1984)

[3] E. Casas and V. Dhamo, Error estimates for the numerical approximation of a quasilinear Neumann problem under minimal regularity of the data. (Submitted).

[4] E. Casas and M. Mateos, Uniform convergence of the FEM. Applications to state constrained control problems. Comp. Appl. Math. 21 (2007) 67-100.

[5] E. Casas and F. Tröltzsch, Optimality conditions for a class of optimal control problems with quasilinear elliptic equations. SIAM J. Control Optim. 48 (2009) 688-718.

[6] P. Ciarlet, The Finite Element Method for Elliptic Problems. North-Holland, Amsterdam (1978).

[7] J. Douglas, Jr. and T. Dupont, A Galerkin method for a nonlinear Dirichlet problem. Math. Comp. 29 (1975) 689-696.

[8] M. Hinze, A variational discretization concept in control constrained optimization: The linear-quadratic case. Comput. Optim. Appl. 30 (2005) 45-61.

[9] I. Hlaváček, Reliable solution of a quasilinear nonpotential elliptic problem of a nonmonotone type with respect to the uncertainty in coefficients. J. Math. Anal. Appl. 212 (1997) 452-466.

[10] I. Hlaváček, M. Kř́žžek and J. Malý, On Galerkin approximations of quasilinear nonpotential elliptic problem of a nonmonotone type. J. Math. Anal. Appl. 184 (1994) 168-189.

[11] L. Liu, M. Křížek and P. Neittaanmäki, Higher order finite element approximation of a quasilinear elliptic boundary value problem of a non-monotone type. Appl. Math. 41 (1996) 467-478.

[12] R. Rannacher and R. Scott, Some optimal error estimates for piecewise finite element approximations. Math. Comp. 38 (1982) 437-445.

[13] P. Raviart and J. Thomas, Introduction à l'Analyse Numérique des Équations aux Dérivées Partielles. Masson, Paris (1983). 